\title{
Intramolecular dynamics. I. Curvilinear normal modes, local modes, molecular anharmonic Hamiltonian, and application to benzene
}

\author{
Yongfeng Zhang, Stephen J. Klippenstein, and R. A. Marcus \\ Arthur Amos Noyes Laboratory of Chemical Physics, California Institute of Technology, \\ Pasadena, California $91125^{\mathrm{a}}$
}

(Received 3 December 1990; accepted 5 February 1991)

\begin{abstract}
The Hamiltonian based on curvilinear normal modes and local modes (CNLM) is discussed using Wilson's exact vibrational Hamiltonian as basis, the CNLM representation diagonalizing only the normal mode block of FG matrix in curvilinear internal coordinates. Using CNLM the kinetic and potential energy operators for benzene are given, including cubic and quartic anharmonicity in the potential energy and cubic and quartic terms in the kinetic energy expansion in curvilinear coordinates. Using symmetrized coordinates and cubic and higher force constants the number and identity of the independent symmetry allowed $\left(A_{1 g}\right)$ such force constants are obtained. The relation to conventional anharmonic force constants is then given and the allowed contributions of the latter are obtained. The results are applied to $\mathrm{CH}$ overtone spectra and intramolecular vibrational dynamics in Part III of this series.
\end{abstract}

\section{INTRODUCTION}

In recent years considerable interest has developed, both experimentally and theoretically, toward understanding vibrational relaxation and intramolecular vibrational redistribution (IVR). It is well known that the properties, rate and mechanism of IVR play a fundamental role in many areas of chemical dynamics. ${ }^{1,2}$ Insight into aspects of RiceRamsperger-Kassel-Marcus RRKM or other treatments of unimolecular reactions, ${ }^{2-4}$ of multiphoton processes, ${ }^{5}$ mode selective processes, ${ }^{6}$ and of overtone-induced reactions ${ }^{7}$ all require elucidation of the IVR behavior. These investigations have been particularly intensified with the introduction and application of lasers and molecular beams to studies of these problems.

In studies of IVR, benzene occupies an interesting position, as a textbook example ${ }^{8,9}$ of a molecule of intermediate size. It shows many of the phenomena exhibited in other intermediate size molecules, such as Fermi resonance, vibration-rotation coupling and radiationless transitions. ${ }^{8-14}$ Several theoretical investigations of IVR of benzene have been performed with the classical trajectory technique, ${ }^{15-19}$ and also with a diagonalization of a model quantum mechanical Hamiltonian ${ }^{20,21}$ or an empirical fitting technique. ${ }^{22}$ The results obtained differ substantially from the more recent $v=3 \mathrm{CH}$ overtone spectrum, the spectrum being much narrower (a factor of 5 or so) than typically calculated values. $^{21}$ The discrepancy may be due either to the dynamical treatment or to the potential energy function used, or to both. A direct quantum-mechanical evaluation for $\mathrm{C}-\mathrm{H}$ overtones, using recent potential energy surface results, should provide more information. The availability of both quadratic force constants and cubic anharmonic force constants for benzene ${ }^{23-33}$ provides one base for the present study. We also introduce some estimated quartic force constants in addition to the quartic terms in the kinetic energy expansion.

\footnotetext{
"Contribution No. 8363
}

On the other hand, as is well known, a basis-set quantum-mechanical study of vibrational spectrum IVR in complicated molecules is often limited by the computational memory and time associated with the use of a large number of zeroth states in high-dimensional systems. However, the use of artificial intelligence (AI) searching methods offers one possible route of performing this evaluation. ${ }^{34}$ This AI search technique is used to find the most important subsets of states from the entire set of zeroth-order states. Once the subset of states is found the characteristics of the vibrational spectrum and dynamics can be analyzed using this reduced block of the full Hamiltonian. ${ }^{34,35}$

In the present series of articles we give such a quantummechanical calculation of vibrational spectra and IVR for $\mathrm{C}-\mathrm{H}$ overtones for benzene for $v_{\mathrm{CH}}=1,2$, and 3 using the AI search technique. In this first paper of the series the curvilinear normal modes and local modes (CNLM) coordinate Hamiltonian to be used to treat benzene is described.

The curvilinear normal mode (CNM) and CNLM coordinate systems are discussed in Sec. II and the Hamiltonian in CNLM coordinates is given. The kinetic couplings for benzene, including the $\mathbf{G}$ matrix and its derivatives, are given in Sec. III. Potential cnergy parameters for benzene, including quadratic, cubic, and quartic force constants, are described in Sec. IV. Relations of cubic terms between nonredundant internal and symmetrized coordinates are discussed in the Appendices.

\section{CURVILINEAR NORMAL MODES AND LOCAL MODES AND HAMILTONIAN}

The conventional theoretical approach for molecular vibrational study is based on the use of rectilinear normal coordinates. ${ }^{8,9,36}$ Rectilinear coordinates, however, need not be the best choice for systems where several degrees of freedom may undergo large-amplitude displacements from the equilibrium configuration. Internal coordinates can be par- 
ticularly useful for treating problems with large-amplitude vibrations, such as occur with higher overtones. ${ }^{23-28,37-42} \mathrm{~A}$ simple polynomial expansion in (curvilinear) internal coordinates is a more accurate representation of the potential than an expansion to the same power of the polynomial in Cartesian coordinates. A number of vibrational and rotational Hamiltonians for polyatomic molecules have been derived in terms of curvilinear normal modes (CNM), such as those given in Refs. 37-42. In the present work curvilinear normal modes and local modes (CNLM) are described and the molecular vibrational Hamiltonian in CNLM is discussed.

We consider a molecule with $M$ vibrational modes, where $M$ is equal to $3 N-6$ or $3 N-5, N$ being the number of atoms in the molecule. When only a subset of the modes is treated, the in-plane modes of benzene, for example, $M<3 N-6$.

\section{A. Curvilinear normal modes and local modes}

The zeroth-order vibrational Hamiltonian of molecular vibrational motion for a harmonic model can be written in terms of displacement coordinates $q$ and their conjugate momenta $\mathbf{p}$ as

$$
\begin{aligned}
H_{0} & =\frac{1}{2} \dot{\mathbf{q}}^{\dagger} \mathbf{G}^{-1}\left(\mathbf{q}_{0}\right) \dot{\mathbf{q}}+\frac{1}{2} \mathbf{q}^{\dagger} \mathbf{F}^{(2)} \mathbf{q} \\
& =\frac{1}{2} \mathbf{p}^{\dagger} \mathbf{G}\left(\mathbf{q}_{0}\right) \mathbf{p}+\frac{1}{2} \mathbf{q}^{\dagger} \mathbf{F}^{(2)} \mathbf{q},
\end{aligned}
$$

where $\mathbf{q}=\left\{q_{1}, q_{2}, \ldots, q_{M}\right\}$ denotes the column vector formed from the nonredundant internal displacement coordinates of the molecule, $\mathbf{p}$ denotes the vector for the momenta conjugate to $\mathbf{q}, \mathbf{G}\left(\mathbf{q}_{0}\right)$ is the customary mass matrix at the equilibrium nuclear configuration $\mathbf{q}_{0}$, denoted later by $\mathbf{G}^{(0)}$, and $F^{(2)}$ is the quadratic force constant matrix.

Curvilinear normal modes $\mathbf{Q}$ describe a particular transformation of $\mathbf{q}$ :

$$
\mathbf{q}=\mathbf{L Q}
$$

or

$$
\mathbf{Q}=\mathbf{S q},
$$

where $\mathbf{Q}=\left\{Q_{1}, Q_{2}, \ldots, Q_{M}\right\}$ denotes a column vector for the CNM, and $\mathbf{L}$ and $\mathbf{S}$ are the transformation matrices. The transformation matrix $\mathbf{L}$ diagonalizes both $\mathbf{G}^{(0)}$ and $\mathbf{F}^{(2)}$ simultaneously, and, in general, the constant factors of $\mathbf{Q}$ are chosen so that the transformed matrix $\mathbf{W}^{-1}\left(\mathbf{q}_{0}\right)$ $=\mathbf{L}^{\dagger} \mathbf{G}^{-1}\left(q_{0}\right) \mathbf{L}$ is a unit matrix, and so that the transformed matrix $K=\mathbf{L}^{\dagger} \mathbf{F}^{(2)} \mathbf{L}$ is diagonal.

This use of CNM for all modes, including $\mathrm{X}-\mathrm{H}$ modes, where $\mathrm{X}=\mathrm{C}, \mathrm{O}, \mathrm{N}$, etc., describes well the small-amplitude molecular vibrations at low vibrational excitations, such as much of the $v=1$ excitation of the $\mathrm{C}-\mathrm{H}$ modes. However, for the excitation of higher $\mathrm{C}-\mathrm{H}$ overtones the CNM description becomes less adequate: The number of states also increases extremely rapidly as the total vibrational energy increases; the densities of vibrational states of benzene for the energy ranges of $\mathrm{C}-\mathrm{H} v=1$ to $v=4$ overtones, for 21 inplane modes, calculated using both direct count and the Whitten-Rabinovitch approximation, ${ }^{2}$ are given in Table $I$, together with the densities of states for some smaller molecules. At $v=3$ the vibrational states have become so closely spaced that they become, almost, a quasicontinuum. Many of these nearly degenerate zeroth-order states are mixed strongly by the anharmonicity of the system, so that each exact vibrational eigenstate would actually be a complicated mixture of many zeroth-order normal mode states and then the CNM no longer provides an adequate description.

For many molecules in which $\mathrm{X}-\mathrm{H}$ overtone studies are of interest ( $\mathrm{X}=\mathrm{C}, \mathrm{O}, \mathrm{N}$, etc.), e.g., water, methane, and benzene, the local mode (LM) description, in which the vibration is largely localized to single $\mathrm{X}-\mathrm{H}$ bonds, provides a simpler picture of the $\mathrm{XH}$ motion. ${ }^{10,12-14}$ For studying such molecules a reasonable procedure is to treat some of the vibrational modes, the $\mathrm{C}-\mathrm{H}$ stretches in benzene, for example, as $\mathrm{LM}$ and to treat the other modes, such as the ring modes and $\mathrm{C}-\mathrm{H}$ bending modes, as CNM. This mixed picture is termed here a curvilinear normal modes and local modes (CNLM) description.

CNM can be determined for a given system. In specifying the CNLM one needs to determine the partitioning be-

TABLE I. Density of vibrational states of benzene and other molecules $\rho_{\text {vib }}$.

\begin{tabular}{lcccc}
\hline \hline Molecule & $\begin{array}{c}\text { Energy } \\
\left(\mathrm{cm}^{-1}\right)\end{array}$ & $\begin{array}{c}\text { Closest } \\
\text { quantum number }\end{array}$ & $\begin{array}{c}\rho_{\mathrm{X}, \mathrm{b}} \\
\text { Direct count }\end{array}$ & $\begin{array}{c}\left.\text { (No. per } \mathrm{cm}^{-1}\right) \\
\text { Whitten-Rabinovitch } \\
\text { approximation }\end{array}$ \\
\hline $\mathrm{H}_{2} \mathrm{O}$ & 10600 & 3 & 0.0050 & 0.0050 \\
& 13830 & 4 & 0.0075 & 0.0080 \\
$\mathrm{C}_{2} \mathrm{H}_{2}$ & 16900 & 5 & 0.0095 & 0.010 \\
& 10000 & 3 & 4.1 & 4.0 \\
$\mathrm{C}_{6} \mathrm{H}_{6}$ & 13000 & 4 & 12.1 & 12.0 \\
$(21$ in-plane modes $)$ & 3000 & 1 & 0.48 & 0.43 \\
& 6000 & 2 & 21.7 & 20.5 \\
$\mathrm{C}_{6} \mathrm{H}_{6}$ & 9000 & 3 & 438 & 422 \\
$(30$ modes $)$ & 12000 & 4 & 5460 & 5310 \\
& 3000 & 1 & 8.8 & 7.0 \\
& 6000 & 2 & 1680 & 1490 \\
\hline \hline
\end{tabular}


TABLE II. Internal and symmetry coordinates of benzene.

\begin{tabular}{|c|c|c|}
\hline & Definition & Description \\
\hline \multicolumn{3}{|c|}{ Internal coordinates } \\
\hline$q_{1}, \ldots, q_{0}$ & $s_{1}, \ldots, s_{6}$ & CH stretching \\
\hline$q_{7, \ldots, q_{12}}$ & $t_{1}, \ldots, t_{6}$ & CC stretching \\
\hline$q_{13}, \ldots, q_{18}$ & $\beta_{1}, \ldots, \beta_{6}$ & $\mathrm{CH}$ in-plane deformation \\
\hline$q_{19}$ & $q_{19}=6^{-1 / 2}\left(\alpha_{1}-\alpha_{2}+\alpha_{3}-\alpha_{4}+\alpha_{5}-\alpha_{6}\right)$ & $B_{1 u}$ ring deformation \\
\hline$q_{200}$ & $\begin{array}{l}q_{20 a}=12^{-1 / 2}\left(2 \alpha_{1}-\alpha_{2}-\alpha_{3}+2 \alpha_{4}\right. \\
\left.\quad-\alpha_{5}-\alpha_{0}\right)\end{array}$ & $E_{2 g}$ ring deformation \\
\hline$q_{20 b}$ & $q_{20 b}=\frac{1}{2}\left(\alpha_{2}-\alpha_{3}+\alpha_{5}-\alpha_{6}\right)$ & $E_{2 g}$ ring deformation \\
\hline $\begin{array}{l}q_{22, \ldots, q_{27}} \\
q_{28}\end{array}$ & $\begin{array}{l}\gamma_{1}, \ldots, \gamma_{6} \\
q_{28}=6^{-1 / 2}\left(\delta_{1}-\delta_{2}+\delta_{3}-\delta_{4}+\delta_{5}-\delta_{6}\right)\end{array}$ & $\begin{array}{l}\text { CH wagging } \\
B_{28} \text { ring deformation }\end{array}$ \\
\hline$q_{29 a}$ & $q_{29_{a}}=\frac{1}{2}\left(-\delta_{1}+\delta_{3}-\delta_{4}+\delta_{6}\right)$ & $E_{28}$ ring deformation ${ }^{n}$ \\
\hline$q_{296}$ & $\begin{array}{l}q_{296}=12-1 / 2\left(-\delta_{1}+2 \delta_{2}-\delta_{3}-\delta_{4}\right. \\
\left.\quad+2 \delta_{5}-\delta_{6}\right)\end{array}$ & $E_{28}$ ring deformation ${ }^{\text {a }}$ \\
\hline \multicolumn{3}{|c|}{ Symmetry coordinates } \\
\hline$A_{12} Q_{1} S_{1}$ & $6^{-1 / 2}\left(t_{1}+t_{2}+t_{3}+t_{4}+t_{5}+t_{6}\right)$ & \\
\hline $\begin{aligned} Q_{2} & S_{2} \\
Q_{28} & S_{3}\end{aligned}$ & $\begin{array}{l}6^{-1 / 2}\left(s_{1}+s_{2}+s_{3}+s_{4}+s_{5}+s_{6}\right) \\
6^{-1 / 2}\left(\beta_{1}+\beta_{2}+\beta_{3}+\beta_{4}+\beta_{5}+\beta_{5}\right)\end{array}$ & \\
\hline$B_{28} Q_{4} S_{4}$ & $-q_{28}$ & \\
\hline$Q_{5} S_{3}$ & $6^{1 / 2}\left(\gamma_{1}-\gamma_{2}+\gamma_{3}-\gamma_{4}+\gamma_{5}-\gamma_{6}\right)$ & \\
\hline$E_{2 g} Q_{0} S_{0, a}$ & $-q_{20 a}$ & \\
\hline$Q_{7} S_{6, b}$ & $-q_{206}$ & \\
\hline$Q_{8} S_{7,0}$ & $12-1 / 2\left(-2 s_{1}+s_{2}+s_{3}-2 s_{4}+s_{5}+s_{6}\right)$ & \\
\hline$Q_{9} S_{7, b}$ & $\frac{1}{2}\left(-s_{2}+s_{3}-s_{5}+s_{6}\right)$ & \\
\hline$Q_{10} S_{8, \alpha}$ & $12^{-1 / 2}\left(-t_{1}+2 t_{2}-t_{3}-t_{4}+2 t_{5}-t_{6}\right)$ & \\
\hline$Q_{11} S_{8, b}$ & $\frac{1}{2}\left(-t_{1}+t_{3}-t_{4}+t_{6}\right)$ & \\
\hline$Q_{12} S_{9, a}$ & $\frac{1}{2}\left(-\beta_{2}+\beta_{3}-\beta_{5}+\beta_{6}\right)$ & \\
\hline$Q_{13} S_{9,6}$ & $12^{-1 / 2}\left(2 \beta_{1}-\beta_{2}-\beta_{3}+2 \beta_{4}-\beta_{5}-\beta_{6}\right)$ & \\
\hline$E_{1,} Q_{14} S_{10, a}$ & $\frac{1}{2}\left(\gamma_{2}+\gamma_{3}-\gamma_{5}-\gamma_{6}\right)$ & \\
\hline$Q_{15} S_{10.6}$ & $12^{-1 / 2}\left(-2 \gamma_{1}-\gamma_{2}+\gamma_{3}+2 \gamma_{4}+\gamma_{5}-\gamma_{6}\right)$ & \\
\hline$A_{24} Q_{16} S_{11}$ & $6^{-1 / 2}\left(\gamma_{1}+\gamma_{2}+\gamma_{3}+\gamma_{4}+\gamma_{5}+\gamma_{6}\right)$ & \\
\hline$B_{14} Q_{17} S_{12}$ & $-q_{19}$ & \\
\hline$Q_{18} S_{13}$ & $6^{-1 / 2}\left(-s_{1}+s_{2}-s_{3}+s_{4}-s_{5}+s_{6}\right)$ & \\
\hline$B_{2 \mu} Q_{19} S_{14}$ & $6^{-1 / 2}\left(-t_{1}+t_{2}-t_{3}+t_{4}-t_{5}+t_{6}\right)$ & \\
\hline$Q_{20} S_{13}$ & $6^{-1 / 2}\left(-\beta_{1}+\beta_{2}-\beta_{3}+\beta_{4}-\beta_{5}+\beta_{6}\right)$ & \\
\hline$E_{2 \mu} Q_{21} S_{10, a}$ & $q_{29 b}$ & \\
\hline$Q_{22} S_{16, b}$ & $-q_{29 a}$ & \\
\hline$Q_{23} S_{17, a}$ & $\frac{1}{2}\left(-\gamma_{2}+\gamma_{3}-\gamma_{5}+\gamma_{6}\right)$ & \\
\hline$Q_{24} S_{17, b}$ & $12^{-1 / 2}\left(-2 \gamma_{1}+\gamma_{2}+\gamma_{3}-2 \gamma_{4}+\gamma_{5}+\gamma_{6}\right)$ & \\
\hline$E_{1 \mu} Q_{2 s} S_{18, a}$ & $\frac{1}{2}\left(\beta_{2}+\beta_{3}-\beta_{5}-\beta_{6}\right)$ & \\
\hline$Q_{26} S_{18, b}$ & $12^{-1 / 2}\left(2 \beta_{1}+\beta_{2}-\beta_{3}-2 \beta_{4}-\beta_{5}+\beta_{6}\right)$ & \\
\hline$Q_{27} S_{19, a}$ & $\frac{1}{2}\left(-t_{1}+t_{3}+t_{4}-t_{6}\right)$ & \\
\hline$Q_{28} S_{19, b}$ & $12^{-1 / 2}\left(t_{1}+2 t_{2}+t_{3}-t_{4} \cdots 2 t_{5}-t_{6}\right)$ & \\
\hline$Q_{29} S_{20, a}$ & $12^{-1 / 2}\left(-2 s_{1}-s_{2}+s_{3}+2 s_{4}+s_{5}-s_{6}\right)$ & \\
\hline$Q_{30} S_{20 . b}$ & $\frac{1}{2}\left(s_{2}+s_{3}-s_{5}-s_{6}\right)$ & \\
\hline
\end{tabular}

'The six $\gamma$ 's and $\delta$ 's are out-of-plane modes. The former is the bending of $\mathrm{CH}$ bond out of the plane of the adjacent $\mathrm{CCC}$ linkage. The latter is the torsion of a CCCC linkage. For example, $\delta_{1}$ is the change in the dihedral angle between the planes determined by $C_{6} C_{1} C_{2}$ and $C_{1} C_{2} C_{3}$ (Ref. 9).

tween these two types of modes. It is convenient in the present work to partition the vibrational modes into $l$ local modes and $M-l$ curvilinear normal modes and to order the components of $\mathbf{Q}$ as $Q_{1}, Q_{2}, \ldots, Q_{l}, Q_{l+1}, \ldots Q_{M}$. The exact definition of CNLM is then the same as in Eq. (2) or (3), but now the transformation matrix $\mathbf{L}$ (or equivalently, $\mathbf{S}$ ) diagonalizes only the normal mode blocks of the matrix $\mathbf{G}^{(0)}$ and $\mathbf{F}^{(2)}$ (as in Fig. 2).

In the present work we consider benzene using the definition, notation, and numbering of the internal coordi- nates, the CNM and the CNLM, given in Tables II and III, and in Fig. 1.

\section{B. Hamiltonian in CNLM}

The quantum mechanical Hamiltonian $H$ of a polyatomic molecule can be written in Hermitian form as ${ }^{9}$

$$
H=\frac{1}{2} \sum_{i, j=1}^{M+3} D^{1 / 4} T_{i} D^{-1 / 2} D_{i j} T_{j} D^{1 / 4}+V(\mathbf{q}),
$$

where the vector $\left(T_{1}, T_{2}, \ldots\right)$ represents the direct sum of the 
TABLE III. Vibrational modes and numbering of benzene.

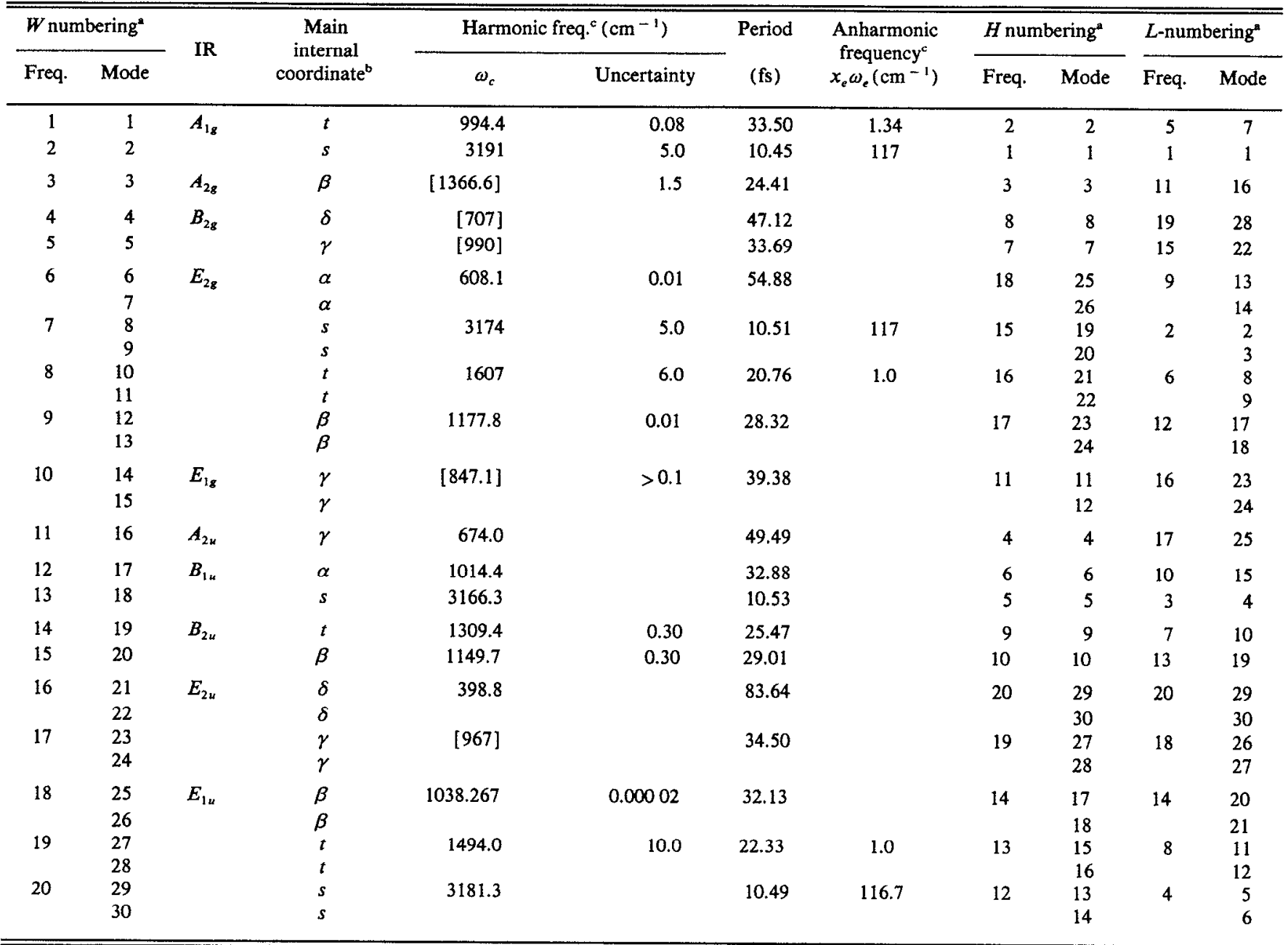

a $W$ numbering-Wilson's numbering, $H$ numbering-Herzberg's numbering, $L$ numbering -numbering used in local-normal-mode coordinates.

${ }^{b}$ The definition of internal coordinates is shown in Table II.

${ }^{\mathrm{c}}$ See Ref. 12, where $[\cdots]$ data obtained indirectly.

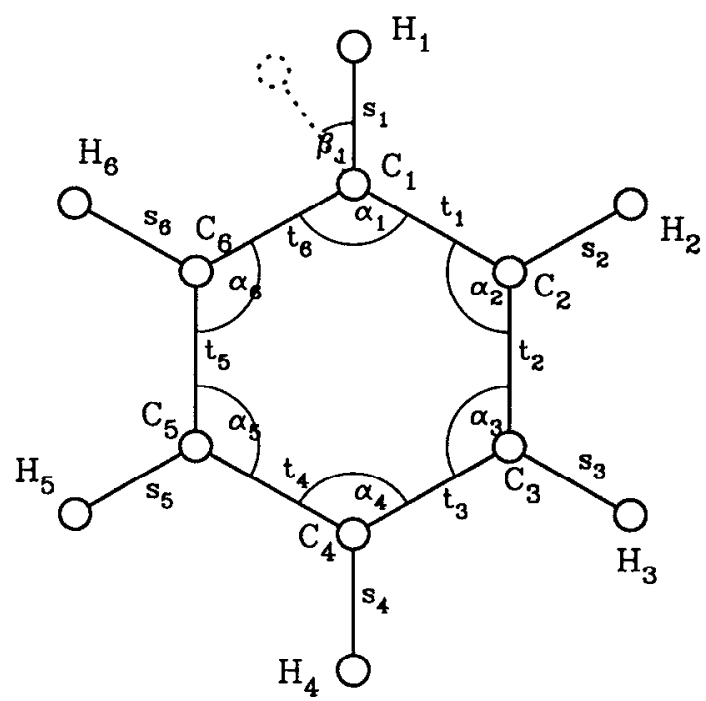

FIG. 1. Internal coordinates of benzene. linear momentum operators $\mathbf{p}$ and the total angular momentum $\mathbf{J}$

$$
\mathbf{T}=\mathbf{p} \oplus \mathbf{J},
$$

and $D=\operatorname{det} \mathbf{D}$. The matrix $\mathbf{D}$ is the direct sum of the mass matrix $\mathbf{G}(\mathbf{q})$ in internal coordinates and inverse of the moment of inertia matrix $\mathbf{I}$, which depends upon Coriolis and other rotational parameters:

$$
\mathbf{D}=\mathbf{G} \oplus \mathbf{I}^{-1} .
$$

In the present initial paper we consider only the vibrational motion and omit, thereby, Coriolis effects. In this case Eq. (4) becomes

$$
H=\frac{1}{2} \sum_{i j=1}^{M} g^{1 / 4} p_{i} g^{-1 / 2} G_{i j}(q) p_{j} g^{1 / 4}+V(q),
$$

where $g=\operatorname{det} \mathbf{G}$ is also a function of q. Expanding the kinetic energy operator in Eq. (7), $H_{K}$, we have 


$$
\begin{aligned}
H_{K}= & \frac{1}{2} \sum_{i j} p_{i} G_{i j} p_{j}+\frac{1}{8} g^{-1} \sum_{i j} G_{i j}\left(p_{i} p_{j} g\right)+\frac{1}{8} g^{-1} \\
& \times \sum_{i j}\left(p_{i} G_{i j}\right)\left(p_{j} g\right)-\frac{5}{32} g^{-2} \sum_{i j}\left(p_{i} g\right) G_{i j}\left(p_{j} g\right),
\end{aligned}
$$

where $\left(p_{i} p_{j} g\right)$ means that the differential operation of $p_{i}$ and $p_{j}$ acts only on $g$. Following an argument analogous to Watson's discussion ${ }^{36}$ the last three terms in Eq. (8) are of a smaller order in $\hbar$ relative to the first terms and are ignored here. We will return to this point in a later paper.

In that case Eq. (7) reduces to

$$
H=\frac{1}{2} \sum_{i, j=1}^{M} p_{i} G_{i j}(\mathbf{q}) p_{j}+V(\mathbf{q}) \text {. }
$$

The matrix $\mathbf{G}$, a matrix function of $\mathbf{q}$, may be expanded about the equilibrium nuclear configuration, $\mathbf{q}=\mathbf{0}$ :

$$
\mathbf{G}(\mathbf{q})=\mathbf{G}^{(0)}+\mathbf{G}^{(1)} \cdot \mathbf{q}+\frac{1}{2 !} \mathbf{G}^{(2)}: \mathbf{q} \mathbf{q}+\cdots,
$$

where $\mathbf{G}^{(i)}$ is the $i$ th derivative of $\mathbf{G}$ with respect to internal coordinates. $\mathbf{G}^{(1)}$ is a vector for each element $G_{i j}$ of the $\mathbf{G}$ matrix and is evaluated at $\mathbf{q}=\mathbf{0}$. It contains $M^{3}$ elements. $\mathbf{G}^{(2)}$, the second derivative of $\mathbf{G}$, is a second-rank tensor for each $G_{i j}$ matrix element and contains $M^{4}$ elements:

$$
\begin{aligned}
& G_{i j, k}^{(1)}=\left[\frac{\partial G_{i j}(\mathbf{q})}{\partial q_{k}}\right]_{\mathbf{q}=0 .} \\
& G_{i j, m n}^{(2)}=\left[\frac{\partial G_{i j}(\mathbf{q})}{\partial q_{m} \partial q_{n}}\right]_{\mathbf{q}=0,}
\end{aligned}
$$

where the indices are $1,2, \ldots, M$ (the indices $M+1$ to $M+3$ in Eq. (4) correspond to J).

When the potential energy term $V(\mathbf{q})$ in Eq. (4) is expanded about the equilibrium nuclear configuration $\mathbf{q}=\mathbf{0}$, we have

$V(\mathbf{q})=\frac{1}{2} \mathbf{F}^{(2)}: \mathbf{q q}+\frac{1}{3 !} \mathbf{F}^{(3)}: \mathbf{q q q}+\frac{1}{4 !} \mathbf{F}^{(4)} \vdots \mathbf{q q q q}+\cdots$,

$\mathbf{F}^{(2)}, \mathbf{F}^{(3)}$, and $\mathbf{F}^{(4)}$ containing the quadratic, cubic and quartic force constants, respectively.

Illustrated by the matrix described in Fig. 2 we have a transformation from internal coordinates $q$ to CNI M $Q$ and also from $\mathbf{p}$ to $\mathbf{P}$,
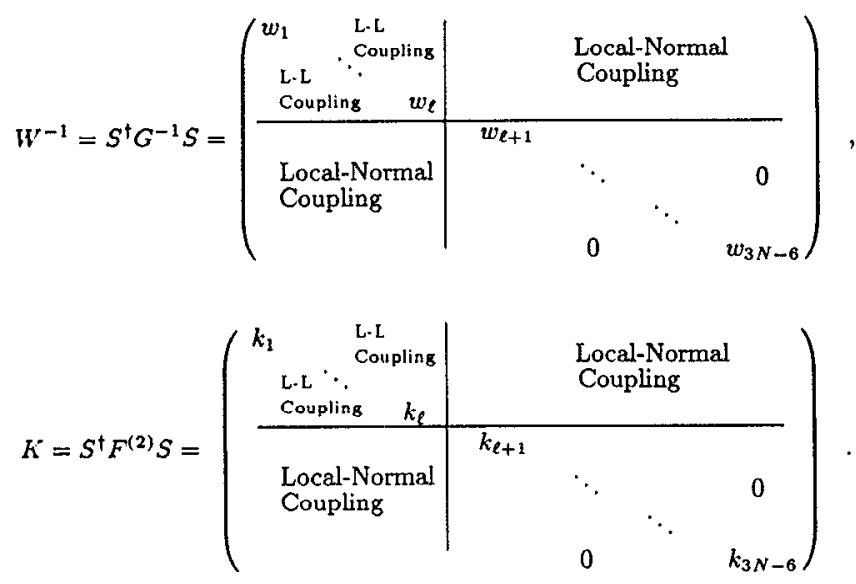

FIG. 2. F and $G$ matrices of local-normal-coordinates.

$$
\begin{aligned}
& q_{i}=\sum_{j} L_{i j} Q_{j}, \\
& p_{i}=\sum_{j}\left(L^{-1}\right)_{j i} P_{j}=\sum_{j} C_{i j} P_{j},
\end{aligned}
$$

where the matrix $\mathbf{C}$ denotes $\left(\mathbf{L}^{-1}\right)^{\dagger}$ and $P_{i}$ is conjugate to $Q_{i}$. Here, neither the matrix $\mathbf{L}$ nor $\mathbf{S}$ is unitary, and therefore $\left(\mathbf{L}^{-1}\right)_{j i} \neq L_{i j}$ and $\mathbf{C} \neq \mathbf{L}$, since the matrix $\mathbf{G F}$ is, in general, not symmetrical, although both $\mathbf{F}$ and $\mathbf{G}$ are symmetrical matrices.

With Eqs. (9), (14), and (15), the Hamiltonian becomes

$$
\begin{aligned}
& H=\frac{1}{2} \mathbf{P}^{\dagger} \mathbf{W}(\mathbf{Q}) \mathbf{P}+V(\mathbf{Q}), \\
& \mathbf{W}(\mathbf{Q})-\mathbf{W}^{(0)}+\mathbf{W}^{(1)} \cdot \mathbf{Q}+\frac{1}{2} \mathbf{W}^{(2)}: \mathbf{Q} \mathbf{Q}+\cdots,
\end{aligned}
$$

where,

$$
\begin{aligned}
& W_{\alpha \beta, \gamma}^{(1)}=\sum_{i, j, k} C_{i \alpha} C_{j \beta} G_{i j, k}^{(1)} L_{k \gamma}, \\
& W_{\alpha \beta, \gamma \delta}^{(2)}=\sum_{i, j, m, n} C_{i \alpha} C_{j \beta} G_{i, m n}^{(2)} L_{m \gamma} L_{n \delta} .
\end{aligned}
$$

While $\mathbf{W}^{(0)}$ is no longer the identity matrix, it contains an $(M-l)-(M-l)$ identity submatrix. In the expansion of $V(\mathbf{Q})$

$$
\begin{aligned}
V(\mathbf{Q})= & \frac{1}{2} \mathbf{K}^{(2)}: \mathbf{Q Q}+\frac{1}{3 !} \mathbf{K}^{(3)}: \mathbf{Q Q Q} \\
& +\frac{1}{4 !} \mathbf{K}^{(4)}: \mathbf{Q Q Q Q}+\cdots,
\end{aligned}
$$

$\mathbf{K}^{(2)}$ is no longer a diagonal matrix, but contains a diagonal block, and $\mathbf{K}^{(3)}$ and $\mathbf{K}^{(4)}$ have the elements,

$$
\begin{aligned}
& K_{\alpha \beta \gamma}^{(3)}=\sum_{i, j, k} L_{i \alpha} L_{j \beta} F_{i j k}^{(3)} L_{k \gamma}, \\
& K_{\alpha \beta \gamma \delta}^{(4)}=\sum_{i, j, m, n} L_{i \alpha} L_{j \beta} F_{i j m n}^{(4)} L_{m \gamma} L_{n \delta} .
\end{aligned}
$$

Since the Morse potential function provides a useful description for local modes, the Morse potential of the $\alpha$ th local mode for $\alpha \leqslant l$, will be used, instead of the terms $K_{\alpha \alpha}^{(2)} Q_{\alpha}^{2} / 2$ !, $K_{\alpha \alpha \alpha}^{(3)} Q_{\alpha}^{3} / 3$ ! and $K_{\alpha \alpha \alpha \alpha}^{(4)} Q_{\alpha}^{4} / 4$ !. We then rewrite the Hamiltonian in Eq. (9) as

$$
H=\sum_{i=1}^{M} h_{i}+H^{\prime},
$$

where the zeroth-order Hamiltonian term $h_{i}$ is

$$
h_{i}= \begin{cases}-\frac{\hbar^{2}}{2 w_{i i}} \frac{\partial^{2}}{\partial Q_{i}^{2}}+D_{i}\left[e^{-2 \alpha_{i} Q_{i}}-2 e^{-\alpha_{i} Q_{i}}\right], & \text { if } i \leqslant l \\ -\frac{\hbar^{2}}{2 w_{i i}} \frac{\partial^{2}}{\partial Q_{i}^{2}}+\frac{1}{2} K_{i i}^{(2)} Q_{i}^{2}, & \text { if } i>l .\end{cases}
$$

The $H^{\prime}$ in Eq. (23) contains, for an intermediate size molecule such as benzene, many terms. Various approximations and models have been employed for $H^{\prime}$ in previous studies. Clarke and Collins neglected $H^{\prime}$ in their classical simulation. ${ }^{19}$ They found that the cubic force constants could not be used since the classical vibrational motion is unstable due to an unbounded potential even for moderate $\mathrm{CH}$ overtones; ${ }^{19}$ Halonen ${ }^{20}$ also omitted cubic and higher force constants, but included one term of the $\mathbf{G}$ matrix, the kinetic coupling between $\mathrm{CH}$ stretch and $\mathrm{CC}$ stretch $G_{\text {st }}^{1}$ (using the 
notation in Ref. 9). Sibert, Reinhardt, and Hynes ${ }^{21}$ introduced two more terms of the $\mathbf{G}$ matrix, $G_{\beta}^{1}$ and $G_{t \beta}^{1}$ and omitted cubic and higher force constants.

In the present work, we consider an extensive quantum calculation and retain many more terms in $H^{\prime}$ :

$$
H^{\prime}=H_{\text {quadratic }}+H_{\text {cubic }}+H_{\text {quartic }}+\cdots \text {. }
$$

The $H_{\text {quadratic }}$ in Eq. (25) arises from the off-diagonal elements of the matrices $W^{(0)}$ and $\mathbf{K}^{(2)}$ in Eqs. (17) and (20), since both $\mathbf{W}^{(0)}$ and $\mathbf{K}^{(2)}$ are no longer diagonal in CNLM. As noted earlier only their normal mode blocks are diagonal. The off-diagonal parts of $\mathbf{W}^{(0)}$ and of $\mathbf{K}^{(2)}$ represent the "stationary kinetic coupling" and the harmonic potential coupling, respectively. Each of these two terms contains the $\mathrm{L}-\mathrm{L}$ (local-local) couplings and L-N (local-normal) coupling as in Fig. 2. Explicitly, we have

$$
H_{\text {quadratic }}=H_{k}^{(2)}+H_{v}^{(2)} \text {, }
$$

where

$$
\begin{aligned}
H_{k}^{(2)} & =\sum_{m=1}^{l} \sum_{n=m+1}^{M} W_{m n}^{(0)} \mathbf{P}_{m} \mathbf{P}_{n}, \\
H_{v}^{(2)} & =\sum_{m=1}^{l} \sum_{n=m+1}^{M} K_{m n}^{(2)} \mathbf{Q}_{m} \mathbf{Q}_{n} .
\end{aligned}
$$

The $H_{\text {cubic }}$ in Eq. (25) involves the first kinetic coupling term in the kinetic energy expansion, i.e., the first derivative of the $\mathbf{G}$ matrix (or $\mathbf{W}^{(1)}$ in CNLM) $H_{k}^{(3)}$, and the cubic anharmonic coupling, $H_{v}^{(3)}$ :

$$
H_{\text {cubic }}=H_{k}^{(3)}+H_{v}^{(3)} \text {, }
$$

where

$$
H_{k}^{(3)}=\frac{1}{2} \sum_{\alpha, \beta, \gamma} W_{\alpha \beta, \gamma}^{(1)} P_{\alpha} Q_{\gamma} P_{\beta},
$$

$\alpha, \beta$, and $\gamma$ are summed over all CNLM's and

$$
H_{v}^{(3)}=\frac{1}{3 !} \sum_{\alpha, \beta, \gamma}^{\prime} K_{\alpha \beta \gamma}^{(3)} Q_{\alpha} Q_{\beta} Q_{\gamma},
$$

and the prime on the summation means that all $K_{\alpha \alpha \alpha}^{(3)} Q_{\alpha}^{3}$ terms for $\alpha \leqslant l$ are excluded, since they were used to construct the Morse potential in Eq. (24).

Similarly, $H_{\text {quartic }}$ involves both the second kinetic coupling, i.e., the second derivative of the $\mathbf{G}$ matrix (or $\mathbf{W}^{(2)}$ in CNLM) $H_{k}^{(4)}$ and the quartic anharmonic coupling, $H_{v}^{(4)}$ :

$$
H_{\text {quartic }}=H_{k}^{(4)}+H_{v}^{(4)} \text {, }
$$

where

$$
H_{k}^{(4)}=\frac{1}{4} \sum_{\alpha, \beta, \gamma, \delta} W_{\alpha \beta, \gamma \delta}^{(2)} P_{\alpha} Q_{\gamma} Q_{\delta} P_{\beta},
$$

and

$$
H_{v}^{(4)}=\frac{1}{4 !} \sum_{\alpha, \beta, \gamma, \delta} K_{\alpha \beta \gamma \delta}^{(4)} Q_{\alpha} Q_{\beta} Q_{\gamma} Q_{\delta},
$$

the prime on the summation again denoting that all $K_{\alpha \alpha \alpha \alpha}^{(4)} Q_{\alpha}^{4}$ terms for $\alpha \leqslant l$ are excluded.

\section{Zeroth-order eigenvectors}

The zeroth-order eigenvectors used in the present work have the form

$$
\phi\left(n_{1}, n_{2}, \ldots n_{M}\right)=\chi_{n_{1}} \chi_{n_{2}} \cdots \chi_{n_{M}},
$$

where $\chi_{n_{j}}$ is a single-mode-eigenvector for mode $j$ with quantum number $n_{j}$. It is an eigenfunction of $h_{j}$, as defined in Eq. (24), with eigenvalue $\epsilon_{n_{j}}$ :

$$
h_{j} \chi_{n_{j}}=\epsilon_{n} \chi_{n_{j}} \text {. }
$$

For NM, the $\chi$ 's are the solutions of the usual harmonic oscillator Schrödinger equation. ${ }^{43}$ For LM, the $\chi$ 's and $\epsilon$ 's are the solutions of the equation for the Morse oscillator: ${ }^{44}$ so

$$
\chi_{n}(y)=N_{n} e^{-y / 2} y^{b / 2} L_{n}^{b}(y),
$$

and

$$
\epsilon_{n}=\left(n+\frac{1}{2}\right) h c \omega-\left(n+\frac{1}{2}\right)^{2} h c \omega x_{e},
$$

where $y=\beta \exp (-\alpha Q), \quad \omega=\alpha(D / 2 \mu)^{1 / 2} /(\pi c), \quad x_{e} \omega$ $=\alpha^{2} h /\left(8 \pi^{2} \mu c\right), \quad \beta=(8 \mu D)^{1 / 2} /(a \hbar)=1 / x_{e}, \quad$ and $b$ $=\beta-2 n-1 . L_{n}^{b}$ is the associated Laguerre polynomials and $N_{n}$ is the normalization constant [ $\alpha(\beta-2 n-1) n ! /$ $\Gamma(\beta-n)]^{1 / 2}, \alpha$ being the Morse constant in Eq. (24).

In the calculation of numerous perturbation terms we can focus on the matrix elements obtained with the singlemode-eigenvectors. The relevant single-mode operators are

$$
Q, Q^{2}, Q^{3}, Q^{4}, P, P^{2}, P Q, Q P, P Q P, P Q^{2}, Q^{2} P \text {, and } P Q^{2} P \text {, }
$$
where $P Q \neq Q P$ and $P Q^{2} \neq Q^{2} P$ due to the noncommutativity of $P$ and $Q$. For the harmonic oscillators, the matrix elements for these operators are readily available. ${ }^{9}$ For the Morse oscillators, the matrix elements for $Q$ and $P$ are given by ${ }^{44-50}$

$$
\begin{aligned}
\langle n+j|P| n\rangle= & i \hbar(-1)^{j+1} N_{n} N_{n+j} \Gamma(\beta-n-1) \\
& \times\left(1-\delta_{j 0}\right) /(2 n !), \quad(j \geqslant 0) \\
\langle n+j|Q| n\rangle= & (-1)^{j+1} \alpha^{-2} N_{n} N_{n+j} \Gamma(\beta-n-j) / \\
& {[j(\beta-2 n-j-1) n !], \quad(j>0) } \\
\langle n|Q| n\rangle=\alpha^{-1}[\ln \beta-\Phi(\beta-2 n-1) & \\
+ & \left.\sum_{j=1}^{n}\left(1-\delta_{n 0}\right) /(\beta-n-j)\right]
\end{aligned}
$$

where

$$
\Phi(z)=d \ln \Gamma(z) / d z .
$$

The expectation values of the other operators can be found using the identity operator, $\Sigma_{n=0}^{\infty}|n\rangle\langle n|=1$, so that, for example, $\left\langle l\left|Q^{2}\right| m\right\rangle$ is given by $\Sigma_{n}\langle l|Q| n\rangle\langle n|Q| m\rangle,{ }^{51}$ and Eqs. (38) and (39) are then used.

\section{KINETIC COUPLING IN BENZENE}

We use the model discussed in Sec. II for the study with the 21 in-plane modes of benzene. Thereby, the coupling between the in-plane modes and the out-of-plane modes and the Coriolis coupling are assumed to be relatively weak for the present purpose. The six $\mathrm{C}-\mathrm{H}$ stretching modes are treated as the LM's and so $l=6$, as in Fig. 2. The remaining 15 modes involve the ring and $\mathrm{C}-\mathrm{H}$ bending motions as the CNM's. 


\section{A. G matrix for benzene}

The mass matrix $\mathbf{G}$ of benzene is well known, the elements of the $\mathbf{G}$ matrix being available both in internal coordinates and symmetry coordinates. ${ }^{9}$ However, what are needed now are not only these elements themselves, but also the derivatives of these elements with respect to the internal coordinates. The usual expressions for these elements in literature, for example in Ref, 9, do not distinguish different $s_{j}, t_{j} \alpha_{j}, \beta_{j}$, etc. (the notation is that given in Fig. 1). Presumably the intention in Ref. 9 and in references cited therein was only to proceed from internal coordinates to conventional (Cartesian) normal coordinates; and hence to use only $\mathbf{G}^{(0)}$ instead of the full coordinate dependent $\mathbf{G}$-matrix $\mathbf{G}(\mathbf{q})$. The element $G_{s_{1}, \beta_{1}}$ was given, for example, as $\sqrt{3} \mu_{c} / 4 t_{0}$ where $t_{0}$ is the $\mathrm{C}-\mathrm{C}$ bond length. ${ }^{9}$ The second derivative of an element, such as $\partial^{2} G T_{s_{1}, \beta_{1}} \partial t_{1} \partial \beta_{1}$, cannot be calculated from these quantities, since it was not specified in the usual tabulation which $\mathrm{C}-\mathrm{C}$ bond displacement $t$ in $\sqrt{3} \mu_{c} / 4 t_{0}$ represents.

Using Wilson's technique the expression of the $\mathbf{G}$ matrix for in-plane displacements, can be derived as explicit functions of internal coordinates. Expressions for nonzero elements $G_{x_{1}, x_{j}^{\prime}}\left(x, x^{\prime}=s, t, \alpha\right.$, and $\left.\beta ; j=1,2, \ldots, 6\right)$ obtained in this way for the $\mathbf{G}$ matrix are given in Table IV. The other nonzero terms $G_{x_{i}, x_{j}}$, with $i=2, \ldots, 6$ can be obtained from the symmetry of the molecule. For example, $G_{t_{2}, \beta_{2}}$ is obtained from $G_{\ell_{1}, \beta_{1}}$ by cycling the subscripts (123456). The exact form of these elements are given in the explicit dependence of the particular coordinates $s, t, \alpha$, and $\beta$ are specified. In particular, $G_{s_{1}, \alpha_{1}}$ equals

$$
\begin{aligned}
G_{s_{1}, \alpha_{1}}= & -\mu_{\mathrm{c}}\left[\tilde{t}_{6}^{-1} \sin \left(\frac{1}{2} \tilde{\alpha}_{1}-\tilde{\beta}_{1}\right)\right. \\
& \left.+\tilde{t}_{1}^{-1} \sin \left(\frac{1}{2} \tilde{\alpha}_{1}+\tilde{\beta}_{1}\right)\right],
\end{aligned}
$$

where $\tilde{t}=t_{0}+t \tilde{\alpha}=\alpha_{0}+\alpha$ and $\tilde{\beta}=\beta_{0}+\beta, t_{0}$ is the bond length of $\mathrm{C}-\mathrm{C}$ bond at equilibrium configuration, $\alpha_{0}=2 \pi / 3$ and $\beta_{0}=0$.

When the internal coordinates for benzene are transformed to CNLM, $\mathbf{G}^{(0)}$ becomes $\mathbf{W}^{(0)}$. For $\mathbf{W}^{(0)}$ there are fifty-two nonzero off-diagonal terms $W_{i j}^{(0)} P_{i} P_{j}$, which form part of the perturbation $H_{\text {quadratic. }}$

\section{B. Derivatives of G matrix}

The first derivatives of the $\mathbf{G}$ matrix with respect to internal coordinates may be obtained either analytically or numerically. However, for the second derivative the former appears to be less practical, since the number of nonzero coefficients $G_{i j, k l}$ defined in Eq. (12) is extremely large. By using a numerical method, namely by writing

$$
\begin{aligned}
G_{i j, m n}^{(2)}= & \frac{1}{\Delta_{m} \Delta_{n}}\left[G_{i j}\left(\ldots, q_{m}+\Delta_{m}, \ldots, q_{n}+\Delta_{n}, \ldots\right)\right. \\
& +G_{i j}\left(\ldots, q_{m}, \ldots, q_{n}, \ldots\right)-G_{i j}\left(\ldots, q_{m}+\Delta_{m}, \ldots, q_{n}, \ldots\right) \\
& \left.-G_{i j}\left(\ldots, q_{m}, \ldots, q_{n}+\Delta_{n}, \ldots\right)\right]
\end{aligned}
$$

convergent results for these second-order kinetic coupling were easily obtained.

We have determined the numbers of primitive independent terms (PIT) for the first and second derivatives of the $\mathbf{G}$ matrix (in symmetrical, conventional normal modes or curvilinear normal modes) by a symmetry study of heterogenous noncommutative spaces: ${ }^{52}$

$$
\begin{gathered}
M_{3}^{\mathrm{PQP}}=\frac{1}{2} M\left(M^{2}+3 M-2\right)+2 M N_{d}-4 N_{d}, \\
M_{4}^{\mathrm{PQQP}}=\frac{1}{4} M\left(M^{3}+6 M^{2}-5 M+2\right) \\
\quad+2 N_{d}\left[(M-2)^{2}-N_{d}+1\right],
\end{gathered}
$$

where the superscripts PQP and PQQP represent spaces spanned by primitive independent basis (PIB) in the first and second derivatives of the $\mathbf{G}$ matrix, respectively, and $N_{d}$ is the number of doubly degenerate irreducible representations (IR) in the $M$ vibrational modes considered. For the in-plane modes of benzene there are 5537 and 66941 PIT's for the first and second derivatives of $\mathbf{G}$ matrix, respectively. However, because of symmetry, the numbers of totally symmetric linear combinations of PIT's, required by symmetry of Hamiltonian, are substantially fewer, namely, 467 and $\mathbf{5 5 7 5}$ for these first and second derivatives of the $\mathbf{G}$ matrix, respectively. ${ }^{52}$

The derivatives of the $\mathbf{G}$ matrix with respect to $\mathrm{CNM}$ or CNLM, which are present in the perturbations $H_{k}^{(3)}$ and $H_{k}^{(4)}$ defined in Eqs. (30) and (33), are obtained by the transformations of Eqs. (18) and (19). The first seventyfive largest elements of the first derivative of the $\mathbf{G}$ matrix for the in-plane modes of benzene, all in unit of $N_{0} \AA^{-1}$ where $N_{0}$ is Avogadro constant, are given in Table V. Here, we consider always derivatives with respect to linear displacements. In obtaining those derivatives, all of angular variables are transformed to corresponding linear displacements. To obtain the derivatives with respect to the curvilinear normal modes $Q_{i}$ listed in Table $\mathrm{V}$ the transformation given in Eq. (18) is used. The $W_{\alpha \beta, \gamma}^{(1)} P_{\alpha} Q_{\gamma} P_{\beta}$ terms of $H_{k}^{(3)}$ can be characterized by types of principal internal coordinate components of the modes $P_{\alpha}, Q_{\gamma}$, and $P_{\beta}$. In principle, there are forty types of the first-order kinetic coupling for in-plane modes of benzene, such as $s s s, \beta s \alpha$, stt, etc., where $s$ denotes a $\mathrm{C}-\mathrm{H}$ stretch, $t$ a C-C stretch, $\alpha$ a CCC angle, and $\beta$ an $\mathrm{HCC}$ angle. However, not all of them are important. For example, the first seventy five largest terms of $H_{k}^{(3)}$ (all expressed in the same units) involve only $\beta s \beta$ and $\beta s t$ type kinetic couplings, while other important terms in $H_{k}^{(3)}$ are tst, $\beta s \alpha$, and $\alpha t t$.

Similarly, for the second-order kinetic couplings $W_{\alpha \beta, \gamma \delta}^{(2)} P_{\alpha} Q_{\gamma} Q_{\delta} P_{\beta}$ for benzene there are one hundred types of the second-order kinetic couplings. The most important types are $\beta$ ss $\beta$ and $\beta$ sst.

\section{POTENTIAL ENERGY PARAMETERS OF BENZENE}

The potential energy function of molecules can be expanded about the equilibrium nuclear configuration in internal coordinates, as in Eq. (13), or in curvilinear normal coordinates, CNM, or in CNLM, as in Eq. (20), or in "Morse coordinates" (Refs. 53 and 54) $\left(1-e^{-\alpha q}\right)$. We adopt the expansions in internal coordinates and CNLM and plan to explore the expansion in Morse coordinates later for the present system. 
TABLE IV. Elements of $g$ matrix for in-plane modes of benzene. ${ }^{a}$

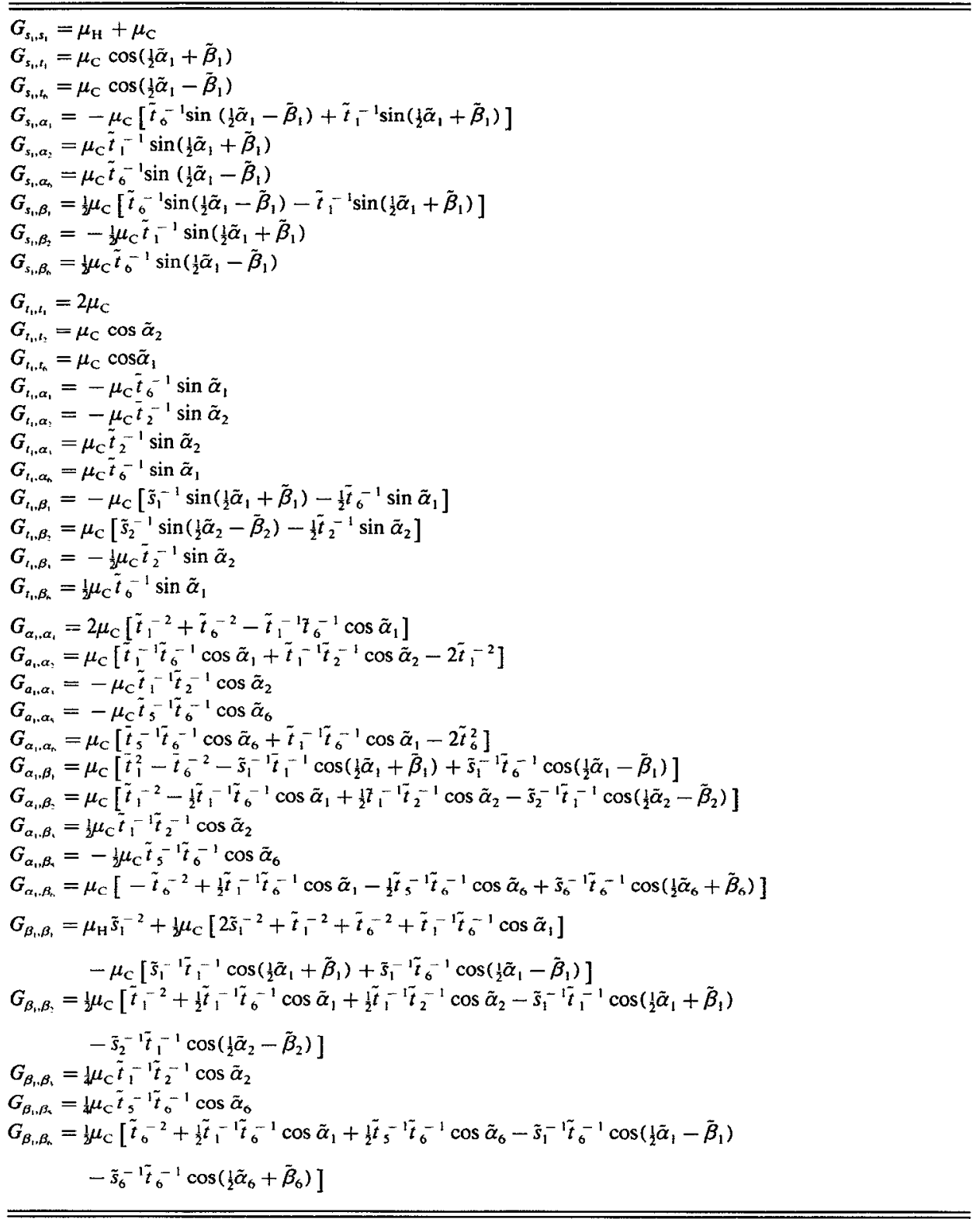

a (1) $\tilde{x}=x_{0}+x$, where $x_{0}$ represents the corresponding values at the equilibrium of nuclear configuration, (e.g., $\alpha_{0}=4 \pi / 3, \beta_{0}=0, t_{0}=$ equilibrium bond length ), $x$ is the displacements, and $x=s_{1}, t_{i}, \alpha_{t}$ or $\beta_{t}$. The definition of $s_{t}, t_{t}, \alpha_{i}$, and $\beta_{i}$ are given in Table II and Fig. $1 . \mu_{\mathrm{H}}=1 / m_{\mathrm{H}}$ and $\mu_{\mathrm{C}}=1 / m_{\mathrm{C}}$.

(2) This table shows only nonvanished elements $G_{x_{1}, x_{j}^{\prime},} x, x^{\prime}=s, t, \alpha$, and $\beta, j=1,2, \ldots, 6$. Other nonvanished elements $G_{x_{r}, x} i=2,3,4,5,6$ can be obtained by symmetry of the molecule.

\section{A. Quadratic force constants of benzene}

The use of computers to perform ab initio calculations of intramolecular potentials, and to refine empirical force constants, has facilitated the theoretical modeling of molecules of ever increasing size. Benzene has recently been the subject of detailed $a b$ initio $^{23,24}$ and semiempirical ${ }^{25-33}$ calculations. Pulay, Fogarasi, and Boggs ${ }^{23}$ (PFB) proposed theoretical quadratic and cubic potential parameters for the ground state of benzene, designated here as the PFB potential. Ozkabak and Goodman ${ }^{26}(\mathrm{OG})$ obtained a harmonic force field from experimental frequencies for $D_{6 h}, D_{3 h}$, and $D_{2 h}$ isotopically labeled benzenes and degenerate mode Coriolis constants, these quadratic force constants being termed here OG potential. To obtain these quadratic constants OG made a correction for the anharmonic contributions to these fundamentals and overtones, when these anharmonicities were available. Ultimately, the OG harmonic potential should be corrected for the effect of the remaining anharmonic contri- 
TABLE V. The first kinetic couplings of in-plane modes of benzene."

\begin{tabular}{|c|c|c|c|c|c|c|c|c|c|c|c|}
\hline$\#$ & Operator & Type of PIT & $W_{i j, k}^{(1)}$ & $\#$ & Operator & Type of PIT & $W_{i, k}^{(1)}$ & $\#$ & Operator & Type of PIT & $W_{i, k}^{(1)}$ \\
\hline 1 & $P_{18} Q_{2} P_{10}$ & $\beta s \beta$ & -1.310 & 26 & $P_{17} Q_{6} P_{12}$ & $\beta_{s t}$ & -0.730 & 51 & $P_{18} Q_{1} P_{18}$ & $\beta s \beta$ & -0.590 \\
\hline 2 & $P_{17} Q_{3} P_{16}$ & $\beta s \beta$ & 1.310 & 27 & $P_{18} Q_{6} P_{11}$ & $\beta s t$ & -0.730 & 52 & $P_{12} Q_{3} P_{11}$ & tst & -0.589 \\
\hline 4 & $P_{19} Q_{6} P_{17}$ & $\beta s \beta$ & 1.150 & 29 & $P_{16} Q_{1} P_{16}$ & $\beta s \beta$ & -0.704 & 54 & $P_{18} Q_{2} P_{9}$ & Bst & 0.498 \\
\hline 5 & $P_{19} Q_{5} P_{18}$ & $\beta s \beta$ & -1.150 & 30 & $P_{16} Q_{3} P_{8}$ & Bst & -0.676 & 55 & $P_{17} Q_{3} P_{9}$ & $\beta s t$ & 0.498 \\
\hline 6 & $P_{16} Q_{6} P_{11}$ & Bst & 1.097 & 31 & $P_{16} Q_{2} P_{9}$ & $\beta s t$ & 0.676 & 56 & $P_{1}, Q_{2} P_{8}$ & $\beta s t$ & -0.498 \\
\hline 10 & $P_{19} Q_{3} P_{11}$ & Bst & 1.031 & 35 & $P_{19} Q_{5} P_{9}$ & Bst & 0.634 & 60 & $P_{11} Q_{1} P_{11}$ & tst & -0.432 \\
\hline 11 & $P_{19} Q_{2} P_{12}$ & $\beta s t$ & -1.031 & 36 & $P_{20} Q_{5} P_{17}$ & $\beta s \beta$ & -0.632 & 61 & $P_{12} Q_{1} P_{12}$ & tst & -0.432 \\
\hline 12 & $P_{20} Q_{0} P_{10}$ & $\beta s \beta$ & -1.011 & 37 & $P_{21} Q_{6} P_{17}$ & $\beta s \beta$ & 0.632 & 62 & $P_{21} Q_{3} P_{20}$ & $\beta s \beta$ & -0.407 \\
\hline 13 & $P_{21} Q_{5} P_{16}$ & $\beta s \beta$ & 1.011 & 38 & $P_{20} Q_{6} P_{18}$ & $\beta_{s} \beta$ & 0.632 & 63 & $P_{17} Q_{2} P_{17}$ & $\beta s \beta$ & 0.406 \\
\hline 14 & $P_{21} Q_{2} P_{19}$ & $\beta s \beta$ & 0.841 & 39 & $P_{21} Q_{5} P_{18}$ & $\beta s \beta$ & 0.632 & 64 & $P_{18} Q_{2} P_{18}$ & $\beta s \beta$ & -0.406 \\
\hline 15 & $P_{20} Q_{3} P_{19}$ & $\beta s \beta$ & -0.841 & 40 & $P_{21} Q_{2} P_{10}$ & Bst & 0.619 & 65 & $P_{10} Q_{5} P_{9}$ & $t s t$ & 0.377 \\
\hline 20 & $P_{20} Q_{1} P_{11}$ & $\beta s t$ & 0.789 & 45 & $P_{21} Q_{3} P_{11}$ & Bst & 0.614 & 70 & $P_{11} Q_{5} P_{8}$ & tst & -0.366 \\
\hline 21 & $P_{21} Q_{1} P_{12}$ & $\beta s t$ & 0.789 & 46 & $P_{11} Q_{3} P_{10}$ & tst & 0.612 & 71 & $P_{11} Q_{6} P_{9}$ & tst & 0.366 \\
\hline 22 & $P_{17} Q_{6} P_{10}$ & $\beta s t$ & 0.753 & 47 & $P_{12} Q_{2} P_{10}$ & tst & -0.612 & 72 & $P_{12} Q_{6} P_{x}$ & $t s t$ & 0.366 \\
\hline 23 & $P_{18} Q_{5} P_{10}$ & Bst & -0.753 & 48 & $P_{21} Q_{4} P_{9}$ & $\beta s t$ & -0.595 & 73 & $P_{12} Q_{5} P_{9}$ & $t s t$ & 0.366 \\
\hline 24 & $P_{14} Q_{1} P_{10}$ & $\beta s t$ & -0.737 & 49 & $P_{20} Q_{4} P_{8}$ & $\beta s t$ & -0.595 & 74 & $P_{20} Q_{1} P_{20}$ & $\beta s \beta$ & -0.347 \\
\hline 25 & $P_{17} Q_{5} P_{11}$ & Bst & 0.730 & 50 & $P_{17} Q_{1} P_{17}$ & $\beta s \beta$ & -0.590 & 75 & $P_{21} Q_{1} P_{21}$ & $\beta s \beta$ & -0.347 \\
\hline
\end{tabular}

- These first kinetic coupling constants are based on the normal modes. All modes are numbered in $L$ numbering (for details see Table II). Only 75 largest $W_{1, k}^{\prime \prime \prime}$ are listed here. All data are in units of $N_{0} \AA^{-1}$, where $N_{0}$ is Avogadro constant. More data for the first kinetic coupling constants, also for the second, are available upon request.

butions to the fundamentals. In our further calculation of the Hamiltonian the OG potential is used.

\section{B. Cubic force constants of benzene}

The only available cubic force constants for benzene are given by the PFB potential in terms of nonredundant internal coordinates. The terms $F_{i j k}^{(3)} q_{i} q_{j} q_{k}$ can be characterized by the types of internal coordinates $q_{i}$ etc., such as sss, st $\alpha$. In general, there are twenty possible types of cubic force constants for in-plane modes of benzene. The PFB potential provides the independent nontrivial cubic force constants. Many other cubic force constants can easily be obtained by cycling the indices of $F_{i j k}^{(3)}$. However, those cubic force constants related to $\alpha$ nonredundant internal coordinates are not as simple to deduce. For practical use of these cubic force constants certain relations among the cubic force constants associated with $s \alpha \alpha, t \alpha \alpha, s s \alpha$, and $t t \alpha$ are needed.

Each of these four types of cubic force constants forms a representation subspace. Since the total Hamiltonian is totally symmetric the numbers of independent quantities in these subspaces are equal to the numbers of $A_{1 g}$ irreducible representations $\Gamma$ in them. From the definition of the internal coordinates used in this work, shown in Table II, $\Gamma_{s}, \Gamma_{t}$, and $\Gamma_{\alpha}$ are six, six, and three dimensional, respectively, and

$$
\begin{aligned}
& \Gamma_{s}=A_{1 g}+B_{1 u}+E_{2 g}+E_{1 u}, \\
& \Gamma_{\imath}=A_{1 g}+B_{2 u}+E_{2 g}+E_{1 u}, \quad \Gamma_{\alpha}=E_{2 g}+B_{1 u},
\end{aligned}
$$

where $\Gamma_{s}$ denotes the representation of the subspace of the six $s^{\prime} s, s_{1}$ to $s_{6}$, etc. The quadratic spaces, $s s, t t$, and $\alpha \alpha$ are 21 ,
21 , and 6-dimensional, respectively. It is readily shown that $^{55-59}$

$$
\begin{aligned}
& \Gamma_{s s}=4 A_{1 g}+2 B_{1 u}+B_{2 u}+4 E_{2 g}+3 E_{1 u}, \\
& \Gamma_{t t}=4 A_{1 g}+B_{1 u}+2 B_{2 u}+4 E_{2 g}+3 E_{1 u}, \\
& \Gamma_{\alpha \alpha}=2 A_{1 g}+E_{2 g}+E_{1 u} .
\end{aligned}
$$

Using direct product and character tables of groups ${ }^{55-59}$ we found that the four types of cubic force constant for benzene, $s \alpha \alpha, t \alpha \alpha$, ss $\alpha$, and $t t \alpha$ have $4,4,6$, and 5 independent quantities, respectively. Other cubic force constants of these types are related to these independent quantities. 'The relations between the independent quantities and other cubic force constants are listed in Table VI. The details for obtaining these relations are discussed in Appendices B and C.

In the AI search calculation in the present series, all of the in-plane cubic force constants given by the PFB potential for benzene are employed.

\section{Estimates and values of quartic force constants}

In previous theoretical calculations for benzene $e^{15-22}$ the quartic couplings (sometimes even cubic couplings) were neglected. The present calculations, details of which are given in Part III of this series, show that these quartic kinetic and potential (where they could be estimated) and kinetic coupling terms make significant contributions to the $v_{\mathrm{CH}}=3$ overtone transition. However, at present most of the quartic force constants of benzene are either not known or have not been estimated. Practical calculations of quartic force constants for some other smaller molecules ${ }^{60-63}$ led to 
TABLE VI. Relations for benzene cubic terms in nonredundant internal coordinates."

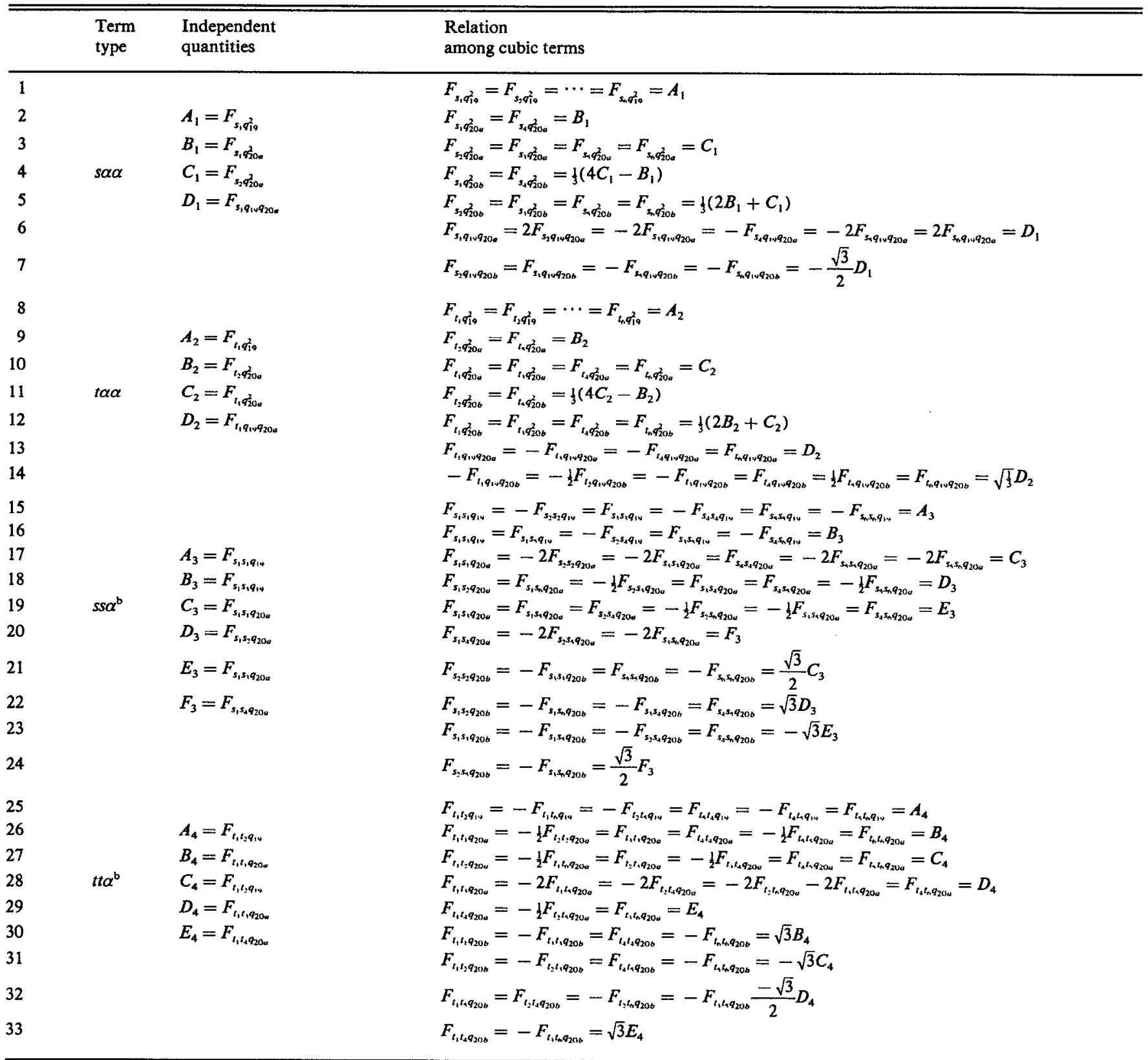

"These cubic force constants are exactly zero:

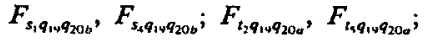

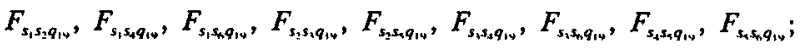

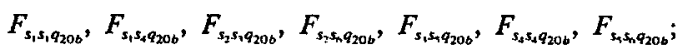

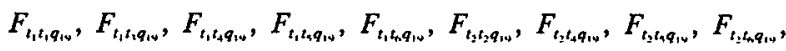

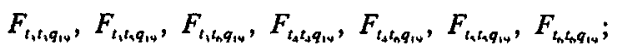

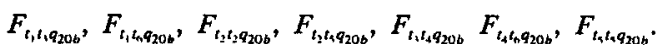

${ }^{\mathrm{b}} F_{s, s, x}=F_{s, p x}$ and $F_{t_{t}, x}=F_{t, f, x}$, where $x=q_{19}, q_{20 a}$, or $q_{20 b}$.

the following inferences: the so-called diagonal quartic force constants, i.e., of the type $F_{x x x x}^{(4)}$, for bond length displacements, are much larger than others, when defined in similar units. In the present initial study we shall confine our atten- tion to the stretching quartic force constants of the type $F_{\text {ssss }}^{(4)}$ and $F_{t u t}^{(4)}$. The actual values of these force constants were obtained approximately using a Morse potential model for the stretching motion. 
TABLE VII. Relation among force constants $F^{(2)}, F^{(3)}$, and $F^{(4)}$.

\begin{tabular}{llccccc}
\hline \hline Molecule & Bond & $F^{(2)}$ & $F^{(3)}$ & $F^{(4)}$ & $F^{(3)} / \sqrt{F^{(2)} F^{(4)}}$ & Ref. \\
\hline HCN & H-C & 3.11 & -5.13 & 4.70 & -1.34 & {$[61]$} \\
& $\mathrm{C} \equiv \mathrm{N}$ & 9.48 & -21.71 & 29.0 & -1.31 & {$[61]$} \\
FH $\cdots \mathrm{H}_{2} \mathrm{O}$ & $\mathrm{F}-\mathrm{H}$ & 10.08 & -80.24 & 514.0 & -1.12 & {$[62]$} \\
& $\mathrm{H} \cdots \mathrm{O}$ & 0.275 & -1.46 & 6.15 & -1.12 & {$[62]$} \\
HNO & $\mathrm{H}-\mathrm{N}$ & 4.36 & -34.10 & 239.0 & -1.06 & {$[63]$} \\
& $\mathrm{N}-\mathrm{O}$ & 12.59 & -88.60 & 604.0 & -1.02 & {$[63]$} \\
& & & & & \\
\hline
\end{tabular}

For the Morse potential the energy $V(x)$ for the coordinate $x$ is

$$
V(x)=D\left[e^{-2 \alpha\left(x-x_{i 1}\right)}-2 e^{-\alpha\left(x-x_{i 1}\right)}\right] .
$$

According to the definition of force constants it is easy to find the relations between $\alpha$ and the quadratic, cubic, and quartic force constants for this system to be

$$
\begin{aligned}
& F^{(3)}=-3 \alpha F^{(2)}, \\
& F^{(4)}=7 \alpha^{2} F^{(2)},
\end{aligned}
$$

where,

$$
F^{(3)} /\left[F^{(2)} F^{(4)}\right]^{1 / 2}=-3 / \sqrt{7} \cong-1.13 .
$$

These approximate relations are in agreement with previous $a b$ initio results, as seen as follows. For benzene $\alpha_{\mathrm{CH}}=1.774 \AA^{-1}$, a value obtained from the anharmonicity of $\mathrm{CH}$ stretching motion, ${ }^{21}$ and $\alpha_{\mathrm{CC}} \approx 1.8 \AA^{-1}$, which is estimated from ethane, ${ }^{50}$ and the values $F_{s_{s} s_{i}}^{(2)}=6.0$ and $F_{t, t,}^{(2)}=7.22$ are given by the PFB potential. ${ }^{23}$ Using Eq. (44) we have

$$
F_{s, s_{1}, s_{1}}^{(3)}=-32 \text { aJ } \AA^{-3}, \quad F_{t_{i}, i_{i}}^{(3)}=-39 \text { aJ } \AA^{-3},
$$

where $i=1, \ldots, 6$. These results are close to $a b$ initio values of the PFB potential, ${ }^{23}-34.0$ and $-40.7 \mathrm{aJ} \AA^{-3}$, respectively.

Equation (46) provides a relation among $F^{(2)}, F^{(3)}$, and $F^{(4)}$. Table VII shows that this approximate relation is reasonably satisfied for some carefully studied small molecules. ${ }^{61-63}$ By using Eq. (46) we obtain

$$
\begin{aligned}
& F_{s, s_{s} s_{1},}^{(4)} \approx 120 \mathrm{aJ} \AA^{-4}, \\
& F_{t, t, t_{t}, t}^{(4)} \approx 150 \mathrm{aJ} \AA^{-4},
\end{aligned}
$$

where $i=1, \ldots, 6$. There is, in Table VII, a trend that the ratio in Eq. (46) is the same within a molecule, for two quite different members of each pair of bonds but differs somewhat from one molecule to another, i.e., from one calculation to another. Perhaps this result reflects an internal consistency within the calculations but the error of a calculation depends on the methods used and the system treated.

\section{SUMMARY}

In summary, a discussion is given here for CNI.M and for the Hamiltonian in this CNLM. The G matrix and potential energy surface for benzene has been discussed. In the further work the results in the present paper will be used to perform an AI search calculation for benzene and calculate (Part III) the $\mathrm{CH}$ spectra for $v_{\mathrm{CH}}=1,2$, and 3 excitations.

\section{ACKNOWLEDGMENTS}

This research was supported by the Caltech Consortium in Chemistry and Chemical Engineering; Founding Members: E. I. du Pont de Nemours and Company, Inc., Eastman Kodak Company, and Minnesota Mining and Manufacturing Company. It is a pleasure to acknowledge also the support of this research by a grant from the National Science Foundation.

\section{APPENDIX A: RELATIONS FOR CUBIC TERMS OF BENZENE IN NONREDUNDANT SYMMETRIZED COORDINATES}

Nonredundant internal coordinates (NRIC) are widely used in literature. ${ }^{23}$ The definition of the NRIC used in this work is given in Table II. However, because of the complicated form of the NRIC involving the $\alpha$ coordinates, the relations between these cubic force constants of benzene in the NRIC are not obviously simple. In this appendix we derive the expressions for these relationships using nonredundant symmetrized coordinates (NRSC). In Appendix B they are derived using redundant internal coordinates (RIC).

In the NRSC system, the $\alpha$ coordinates are symmetrized and other in-plane modes, $s, t$, and $\beta$, still use internal coordinates. There are $63,63,36$, and 36 possible cubic force constants in the $s \alpha \alpha, t \alpha \alpha, s s \alpha$, and $t$ t $\alpha$ subspaces. However, as is shown in Sec. IV B there are only $4,4,6$, and 5 independent quantities, respectively. Others can be a linear combination of the independent quantities in the corresponding subspace.

We can use nonredundant symmetrized rectilinear or curvilinear coordinates to find the relationships between independent quantities and other cubic force constants. These relationships are simpler than those in Table VI. We consider the $s \alpha \alpha$ and $t \alpha \alpha$ types of cubic force constants as examples.

\section{1. $s$ coordinates}

In Table II six relations between the six symmetric $s$ type coordinates $q^{s}$, denoted in Table II by $S_{2}$, $S_{7 a}, S_{7 b}, S_{13}, S_{20 a}$, and $S_{20 b}$ using Wilson's numbering, ${ }^{9,13}$ and the six internal coordinates $s_{i}$ for $\mathrm{CH}$ stretching are given. They form a $6 \times 6$ matrix. The $s_{i}(i=1, \ldots, 6)$ are written as linear combinations of the symmetrized coordinates $q^{3}$ in Eq. (A1). The coefficients relating the six symmetrized coordinates $q^{s}$ to the $s_{i}$ is obtained by transposing the abovementioned matrix. 


\begin{tabular}{|c|c|c|c|c|c|c|c|c|}
\hline \multirow{3}{*}{$s_{1}$} & \multirow{11}{*}{$=$} & 1 & -2 & 0 & -1 & -2 & 0 & \\
\hline & & $\sqrt{6}$ & $\sqrt{12}$ & & $\sqrt{6}$ & $\sqrt{12}$ & & $q_{a \mathrm{lg}}^{s}$ \\
\hline & & 1 & 1 & 1 & 1 & -1 & 1 & \\
\hline \multirow[t]{2}{*}{$s_{2}$} & & $\sqrt{6}$ & $\overline{\sqrt{12}}$ & $\overline{2}$ & $\overline{\sqrt{6}}$ & $\overline{\sqrt{12}}$ & $\overline{2}$ & $q_{e 2 g a}^{s}$ \\
\hline & & 1 & 1 & 1 & -1 & 1 & 1 & \\
\hline$s_{3}$ & & $\overline{\sqrt{6}}$ & $\overline{\sqrt{12}}$ & $\overline{2}$ & $\overline{\sqrt{6}}$ & $\overline{\sqrt{12}}$ & 2 & $q_{e 2 g b}^{2}$ \\
\hline$s_{4}$ & & $\frac{1}{\sqrt{6}}$ & $\frac{-2}{\sqrt{12}}$ & 0 & $\frac{1}{\sqrt{6}}$ & $\frac{2}{\sqrt{12}}$ & 0 & $q_{b 1_{k}}^{s}$ \\
\hline$s_{5}$ & & 1 & 1 & 1 & -1 & 1 & 1 & $q_{p \mid u}^{s}$ \\
\hline & & $\sqrt{6}$ & $\sqrt{12}$ & 2 & $\overline{\sqrt{6}}$ & $\overline{\sqrt{12}}$ & $\overline{2}$ & \\
\hline$s_{6}$ & & 1 & 1 & 1 & 1 & -1 & -1 & $q_{e 1 u b}^{s}$ \\
\hline 10 & & $\sqrt{6}$ & $\overline{\sqrt{12}}$ & $\overline{2}$ & $\sqrt{6}$ & $\overline{\sqrt{12}}$ & 2 & \\
\hline
\end{tabular}

\section{2. $\alpha \alpha$ coordinates}

There are six redundant $\alpha$ coordinates $\alpha_{1}$ to $\alpha_{6}$. In the $\alpha$ space there three coordinates in NRIC, $q_{19}$ and $q_{20 a, b}$. We introduce the coordinates,

$$
\begin{aligned}
& q_{20}^{+}=\frac{1}{\sqrt{2}}\left(q_{20 a}+i q_{20 b}\right), \\
& q_{20}^{-}=\frac{1}{\sqrt{2}}\left(q_{20 a}-i q_{20 b}\right) .
\end{aligned}
$$

The six members of the basis set in the corresponding quadratic $\alpha \alpha$-subspace can be combined into four irreducible representations: two $A_{1 g}$, one $E_{2 g}$ and one $E_{1 u}$, as seen in Sec. IV $B$. The six symmetrized quadratic basis elements in the $\alpha \alpha$ space are denoted by $\theta_{1}$ to $\theta_{6}$ :

$$
\begin{aligned}
& \theta_{1}=\theta_{a 1 g, 1}^{\alpha \alpha}=q_{19} q_{19}, \quad \theta_{2}=\theta_{a 1 g, 2}^{\alpha \alpha}=q_{20}^{+} q_{20}^{-}, \\
& \theta_{3}=\theta_{e 2 g, a}^{\alpha \alpha}=-\frac{\sqrt{3}}{2}\left(q_{20}^{+} q_{20}^{+}+q_{20}^{-} q_{20}^{-}\right), \\
& \theta_{4}=\theta_{e 2 g, b}^{\alpha \alpha}=\frac{1}{i} \sqrt{\frac{3}{8}}\left(q_{20}^{+} q_{20}^{+}-q_{20}^{-} q_{20}^{-}\right), \\
& \theta_{5}=\theta_{e 1 u, a}^{\alpha \alpha}=\frac{1}{\sqrt{2}} q_{19}\left(q_{20}^{+}+q_{20}^{-}\right), \\
& \theta_{6}=\theta_{e 1 u, b}^{\alpha \alpha}=\frac{1}{\sqrt{2}} q_{19}\left(q_{20}^{+}-q_{20}^{-}\right) .
\end{aligned}
$$

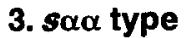

As seen in Sec. IV B, there are four independent quantities or four $A_{1 g}$ linear combinations in the s $\alpha \alpha$ subspace, among others of a different symmetry species. The contribution to the potential energy from the sa $\alpha$ subspace, denoted by $V_{s \alpha \alpha}$, may be written in two equivalent ways, in NRIC and in NRSC, respectively:

$V_{s \alpha \alpha}=\frac{1}{3 !} \sum_{j=1}^{6} \sum_{k=1}^{6} F_{s_{j} \theta_{k}} s_{j} \theta_{k}=\frac{1}{3 !} \sum_{i=1}^{4} F_{i}^{s \alpha \alpha} \Phi_{i}^{s \alpha \alpha}$,

where $\Phi_{i}^{s \alpha \alpha}$ is the ith $A_{18}$ component in the s $\alpha \alpha$ subspace, and
$F_{i}^{s \alpha \alpha}$ is its associated cubic force constant. Since $\theta_{1}$ and $\theta_{2}$, defined in Eq. (A1), are already totally symmetric $\left(A_{1 g}\right)$, only the $A_{1 g}$ component of the six $s_{i}$ coordinates makes a nonzero contribution to the Hamiltonian $s \alpha \alpha$ terms. It can be seen from Eq. (A1) that the coefficient for the $q_{a 1 g}^{s}$ component is the same for all $s_{i}$, namely $1 / \sqrt{ } 6$. We have, therefore,

$$
\begin{aligned}
& \Phi_{1}^{s \alpha \alpha}=\frac{1}{\sqrt{6}}\left(s_{1}+s_{2}+s_{3}+s_{4}+s_{5}+s_{6}\right) q_{19} q_{19}, \\
& \Phi_{2}^{s \alpha \alpha}=\frac{1}{\sqrt{6}}\left(s_{1}+s_{2}+s_{3}+s_{4}+s_{5}+s_{6}\right) q_{20}^{+} q_{20}^{-} .
\end{aligned}
$$

Equation (5) provides two independent quantities in Eq. (A4) which we shall denote by $F_{1}^{s \alpha \alpha}=\sqrt{6} A_{i}^{\prime}$ and $F_{2}^{s \alpha \alpha}=\sqrt{6} B_{1}^{\prime}$, respectively. Then

$$
\begin{aligned}
V_{s \alpha \alpha} & =\frac{1}{3 !}\left[\sum_{j=1}^{6} F_{s ; q_{19} q_{19}} s_{j} q_{19} q_{19}+\sum_{j=1}^{6} F_{s, q_{20}^{+} q_{20}} s_{j} q_{20}^{+} q_{20}^{-}\right]+\cdots \\
& =\frac{1}{3 !}\left[F_{1}^{s \alpha \alpha} \Phi_{1}+F_{2}^{s \alpha \alpha} \Phi_{2}\right]+\cdots
\end{aligned}
$$

Shown only by dots are the contributions from $\Phi_{3}^{\text {sac }}$ and $\Phi_{4}^{\text {saa }}$ (see below). Substituting Eq. (A5) into (A6) and comparing both sides we have

$$
F_{s, q_{19} q_{19}}=A_{1}^{\prime}, \quad F_{s f_{20}^{+} q_{20}^{-}}=B_{1}^{\prime}, \quad(j=1 \cdots 6) .
$$

Similarly, there are two other $A_{1 g}$ linear combinations, $\Phi_{3}^{s \alpha \alpha}$ and $\Phi_{4}^{s \alpha \alpha}$ generated from the $E_{2 g}$ and $E_{1 u}$ components in $s$ and $\alpha \alpha$ spaces, respectively. We choose the other two independent quantities as $F_{s_{1}\left(q_{20}^{+} q_{20}^{+}+q_{20}^{-} q_{20}^{-}\right)}$and $F_{s_{1} q_{1 y}\left(q_{20}^{+}+q_{20}^{-}\right)}$and denote them by $C_{1}^{\prime}$ and $D_{1}^{\prime}$, respectively. The other cubic constants in this s $\alpha \alpha$ subspace are related to them. This space of four states will be denoted by $\left\{\phi_{a}, \phi_{b}, \phi_{a}^{\prime}, \phi_{b}^{\prime}\right\} . \phi_{a}$ is the $a$ component of the $E_{2 g}$ pair or of the $E_{1 u}$ pair, with a similar remark applied to the other $\phi$ 's. It is readily shown using group theory that the $A_{1 g}$ lincar combination generated from components of two $E_{2 g}$ (or $E_{1 u}$ ) states spanned by $\left\{\phi_{a}, \phi_{b}\right\}$ and $\left\{\phi_{a}^{\prime}, \phi_{b}^{\prime}\right\}$, respectively, is 


$$
\psi_{A_{1,}}=(1 / \sqrt{2})\left(\phi_{a} \phi_{a}^{\prime}+\phi_{b} \phi_{b}^{\prime}\right)
$$

Following similar discussion above for $\Phi_{1}^{s \alpha \alpha}$ and $\Phi_{2}^{s \alpha \alpha}$, using the above expression (A8) and the coefficients given in Eq. (A1) we have

$$
\begin{aligned}
& F_{s_{1}\left(q_{20}^{+} q_{20}^{+}+q_{20}^{-} q_{20}^{-}\right)}
\end{aligned}
$$

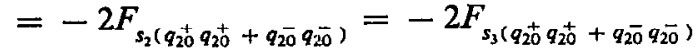

$$
\begin{aligned}
& =F_{s_{4}\left(q_{20}^{+} q_{20}^{+}+q_{20}^{-} q_{20}^{-}\right)}=-2 F_{s_{5}\left(q_{20}^{+} q_{20}^{+}+q_{20}^{-} q_{20}^{-}\right)} \\
& =-2 F_{s_{6}\left(q_{20}^{+} q_{20}^{+}+q_{20}^{-} q_{20}\right)}=C_{1}^{\prime} \text {, } \\
& F_{s_{2}\left(q_{20}^{+} q_{20}^{+}-q_{20}^{-} q_{20}^{-}\right)}=-F_{s_{3}\left(q_{20}^{+} q_{20}^{+}-q_{20}^{-} q_{20}^{-}\right)}=F_{s_{5}\left(q_{20}^{+} q_{20}^{+}-q_{20} \overline{q_{20}}-\overline{0}\right.} \\
& =-F_{s_{n}\left(q_{20}^{+} q_{20}^{+}-q_{20}^{-} q_{20}^{-}\right)}=\frac{\sqrt{3}}{2} C_{1}^{\prime}, \\
& F_{s_{1} q_{19}\left(q_{20}^{+}+q_{20}\right)}=2 F_{s_{2} q_{19}\left(q_{20}^{+}+q_{20}\right)}=-2 F_{s_{3} q_{19}\left(q_{20}^{+}+q_{20} \overline{0}\right.} \\
& =-F_{s_{4} q_{19}\left(q_{20}^{+}+q_{20}\right)}=-2 F_{s_{9} q_{19}\left(q_{20}^{+}+q_{20}^{-}\right)} \\
& =2 F_{s_{6} q_{19}\left(q_{20}^{+}+q_{20}\right)}=D_{i}^{i} \text {, } \\
& F_{s_{2} q_{19}\left(q_{20}^{+}-q_{20}\right)}=F_{s_{3} q_{19}\left(q_{20}^{+}-q_{20}^{-}\right)}=-F_{s_{3} q_{19}\left(q_{20}^{+}-q_{20}\right)} \\
& =-F_{s_{s_{1}} q_{19}\left(a_{20}^{+}-q_{20}^{-}\right)}=-\frac{\sqrt{3}}{2} D_{1}^{\prime} \text {. }
\end{aligned}
$$

It is also follows from the combination coefficients and irreducible representations given in (A1) and (A3) that

$$
\begin{aligned}
F_{s_{1}\left(q_{20}^{+} q_{20}^{+}-q_{20} q_{20}\right)} & =F_{s_{4}\left(q_{20}^{+} q_{20}^{+}-q_{20} q_{20}\right)}=F_{s_{1} q_{19}\left(q_{20}^{+}-q_{20}^{-}\right)} \\
& =F_{s_{4} q_{14}\left(q_{20}^{+}-q_{20}^{-}\right)}=0 .
\end{aligned}
$$

\section{4. $t \alpha \alpha$ type}

The relation between six $t$-type internal coordinates and symmetrized coordinates, similarly to that given in Eq. (A1), can be obtained from Table II. In the same way as that for the $s \alpha \alpha$ subspace, we have

$$
\begin{aligned}
& -2 F_{t_{1}\left(q_{20}^{+} q_{20}^{+}+q_{20} q_{20}\right)} \\
& =F_{t_{2}\left(q_{20}^{+} q_{20}^{+}+q_{20}^{-} q_{20}^{-}\right)}=-2 F_{t_{3}\left(q_{20}^{+} q_{20}^{+}+q_{20}^{-} q_{20}^{-}\right)} \\
& =-2 F_{t_{4}\left(q_{20}^{+} q_{20}^{+}+q_{20}^{-} q_{20}^{-0}\right)}=F_{t_{1}\left(q_{20}^{+} q_{20}^{+}+q_{20}^{-} q_{20}^{-}\right)} \\
& =-2 F_{t_{0}\left(q_{20}^{+} q_{20}^{+}+q_{20} q_{20}\right)}=C_{2}^{\prime} \text {, } \\
& F_{t_{1}\left(q_{20}^{+}-q_{20}^{+}-q_{20}^{-} q_{20}^{-}\right)} \\
& =-F_{t_{3}\left(q_{20}^{+} q_{20}^{+}-q_{20}^{-} q_{20}^{-}\right)}=F_{t_{4}\left(q_{20}^{+} q_{20}^{+}-q_{20}^{-} q_{20}^{-}\right)} \\
& =-F_{t_{6}\left(q_{20}^{+} q_{20}^{+}-q_{20}^{-} q_{20}^{-}\right)}=-\frac{\sqrt{3}}{2} C_{2}^{\prime} \text {, } \\
& F_{t_{1} q_{14}\left(q_{20}^{+}+q_{20}^{-}\right)}=-F_{t_{3} q_{14}\left(q_{20}^{+}+q_{20}^{-}\right)}=-F_{t_{4} q_{14}\left(q_{20}^{+}+q_{20}\right)} \\
& =F_{t_{h} q_{14}\left(q_{20}^{+}+q_{20}^{-}\right)}=-\frac{\sqrt{3}}{2} D_{2}^{\prime} \\
& 2 F_{t_{1} q_{14}\left(q_{20}^{+}-q_{20}\right)}=F_{t_{2} q_{14}\left(q_{20}^{+}-q_{20}^{-}\right)}=2 F_{t_{3} q_{14}\left(q_{20}^{+}-q_{20}\right)} \\
& =-2 F_{t_{4} q_{14}\left(q_{20}^{+}-q_{20}\right)}=-F_{t_{5} q_{14}\left(q_{20}^{+}-q_{20}\right)} \\
& =-2 F_{t_{1} q_{14}\left(q_{20}^{+}-q_{20}\right)}=D_{2}^{\prime} \text {, }
\end{aligned}
$$

where $F_{t_{1} q_{14} q_{14}}, F_{t_{1} q_{20}^{+} q_{20}^{-}}, F_{t_{2}\left(q_{20}^{+} q_{20}^{+}+q_{20}^{-} q_{20}^{-}\right)}$, and $F_{t_{2} q_{14}\left(q_{20}^{+}-q_{20}^{-}\right)}$ are chosen as independent quantities and noted by $A_{2}^{\prime}, B_{2}^{\prime}$, $C_{2}^{\prime}$, and $D_{2}^{\prime}$, respectively.

Other types of cubic terms also can be treated similarly.

\section{APPENDIX B: RELATIONS FOR CUBIC TERMS OF BENZENE IN NONREDUNDANT INTERNAL COORDINATES}

Four different types of cubic force constants, $s \alpha \alpha, s s \alpha$, $t \alpha \alpha$, and $t t \alpha$ are discussed, respectively.

\section{1. sa $\alpha$ type}

There are 216 force constants for the terms $s_{k} \alpha_{i} \alpha_{j}$ in RIC of benzene. However, a careful examination shows that only 13 of these are independent cubic force constants in RIC for this subspace. These independent terms, represented by structure plots, are given in Fig. 3(a) where a bar represents the position of $s_{k}$ (without loss of generality we choose $k=1$, i.e., the first position), and the two asterisks indicate the positions of $\alpha_{i}$ and $\alpha_{j}$. The corresponding cubic force constants are denoted by $F_{1}^{s}, F_{2}^{s}, \ldots, F_{13}^{s}$, respectively. Any cubic force constant of type s $\alpha \alpha$ is equal to one of these $F_{m}^{s}$ 's. For example, $s_{1} \alpha_{2} \alpha_{2}$ is represented by the fifth structure in Fig. 3(a) and $s_{2} \alpha_{1} \alpha_{6}$ is equivalent to the seventh structure in Fig. 3(a). Therefore $F_{s_{1} \alpha_{2} \alpha_{2}}=F_{5}^{s}$ and $F_{s_{2} \alpha_{1} \alpha_{o}}$ $=F_{7}^{s}$, respectively. In this way the assignments of the $s_{1} \alpha_{i} \alpha_{j}$ and the $s_{2} \alpha_{i} \alpha_{j}$ are obtained and given in Table VIII. The assignment for other $s_{k}$ 's can be obtained similarly.

By using the definition of $q_{19}$ given in Table II and Table VIII, we obtain

$$
\begin{aligned}
F_{s_{1} q_{19}^{2}}= & \frac{1}{6}\left[F_{1}^{s}-4 F_{2}^{s}+4 F_{3}^{s}-2 F_{4}^{s}+2 F_{s}^{s}+2 F_{6}^{s}-4 F_{7}^{s}\right. \\
& \left.-4 F_{8}^{s}+4 F_{9}^{s}+2 F_{10}^{s}+2 F_{11}^{s}-4 F_{12}^{s}+F_{13}^{s}\right] .
\end{aligned}
$$

Similarly, we obtained the same expression for $F_{s_{1} q_{19}^{2}}$ for $i=2,3, \ldots, 6$. The first relationship in Table VI is thereby established.

By using the definition of $q_{20 a}$ in Table II, we obtain two sets of equivalent cubic force constants:

(a)
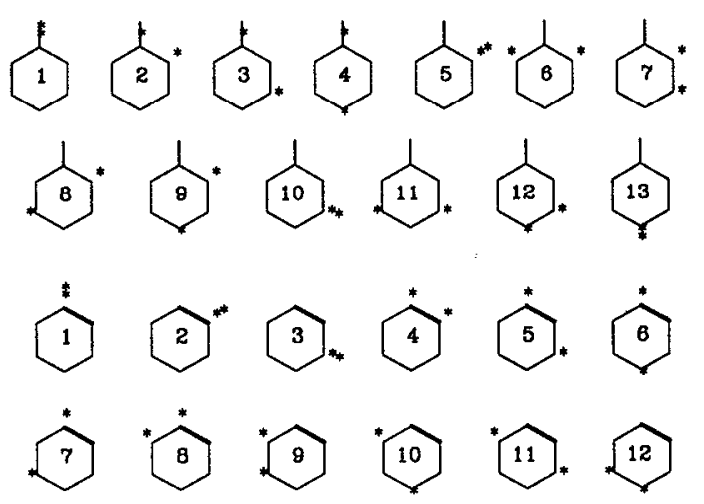

FIG. 3. Independent terms in cubic force constants related to $\alpha$ internal coordinates in NRIC: (a) sa $\alpha$ type, where $s$ is fixed as $s_{1}$ and the positions of $\alpha$ are represented by $*$. For example, the sixth means the term $s_{1} \alpha_{2} \alpha_{6}$. (b) $t \alpha \alpha$ type, where $t$ is fixed as $t_{1}$ and the positions of $\alpha$ are represented by $*$. For example, the sixth means the term $t_{1} \alpha_{1} \alpha_{4}$. 


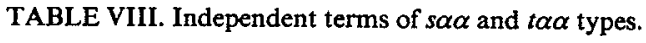

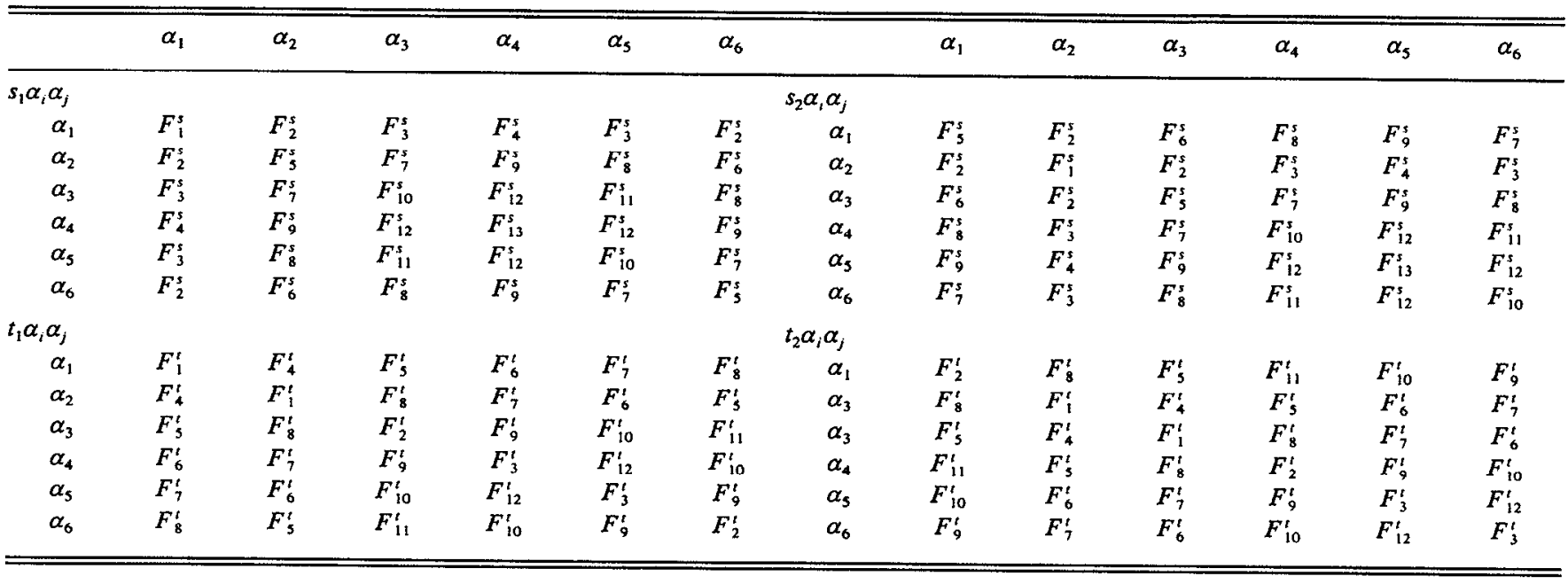

$$
\begin{aligned}
F_{s_{f} q_{20 a}^{2}}=B_{1}= & \frac{1}{6}\left[2 F_{1}^{s}-4 F_{2}^{s}-4 F_{3}^{s}+4 F_{4}^{s}+F_{5}^{s}+F_{6}^{s}\right. \\
& +2 F_{7}^{s}+2 F_{8}^{s}-4 F_{9}^{s}+F_{10}^{s}+F_{11}^{s} \\
& \left.-4 F_{12}^{s}+2 F_{13}^{s}\right],
\end{aligned}
$$

for $i=1,4$ and

$$
\begin{aligned}
F_{s_{1} q_{20 a}^{2}}=C_{1}= & \frac{1}{12}\left[F_{1}^{s}-2 F_{2}^{s}-2 F_{3}^{s}+2 F_{4}^{s}+5 F_{5}^{s}-4 F_{6}^{s}\right. \\
& -8 F_{7}^{s}+10 F_{8}^{s}-2 F_{9}^{s}+5 F_{10}^{s}-4 F_{11}^{s} \\
& \left.-2 F_{12}^{s}+F_{13}^{s}\right],
\end{aligned}
$$

for $i=2,3,5,6$.

By using the definition of $q_{20 b}$ in Table II we have

$$
F_{s, q_{20 b}^{2}}=\frac{1}{2}\left[F_{s}^{s}-F_{6}^{s}-2 F_{7}^{s}+2 F_{8}^{s}+F_{10}^{s}-F_{11}^{s}\right]
$$

for $i=1,4$ and

$$
\begin{aligned}
F_{s_{i} q_{20 b}^{2}}= & \frac{1}{4}\left[F_{1}^{s}-2 F_{2}^{s}-2 F_{3}^{s}+2 F_{4}^{s}+F_{s}^{s}\right. \\
& \left.+2 F_{8}^{s}-2 F_{9}^{s}-2 F_{12}^{s}+F_{13}^{s}\right],
\end{aligned}
$$

for $i=2,3,5,6$.

Combining Eqs. (B1)-(B4), we have the relations between cubic force constants in NRIC:

$$
\begin{aligned}
& F_{s, q_{20 b}^{2}}=\frac{1}{3}\left(4 C_{1}-B_{1}\right), \quad j=1,4 \\
& F_{s, q_{20 b}^{2}}=\frac{1}{3}\left(2 B_{1}+C_{1}\right), \quad j=2,3,5,6
\end{aligned}
$$

as shown in Eqs. 2 to 5 in Table VI.

Other relations for $F_{s, q_{14} q_{20 a}}$ and $F_{s, q_{14} q_{20 b}}$ can be established similarly.

\section{2. toc type}

Here, $t$ represents CC stretching as in Fig. 1 and Table II. There 216 terms $t_{k} \alpha_{i} \alpha_{j}$ in RIC. However, a careful examination shows that only 12 of them are independent. These are shown in Fig. 3(b). The corresponding cubic force constants are denoted by $F_{1}^{t}, F_{2}^{t}, \ldots, F_{12}^{t}$, respectively. Any cubic force constant of type t $\alpha \alpha$ is equal to one of $F_{m}^{i}$. The assignment of $F_{t_{1} \alpha_{\alpha_{j}} \alpha_{j}}$ and $F_{t_{2} \alpha_{j} \alpha_{j}}$ are given in Table VIII, and the assignment for other $t_{k}$ can be obtained similarly.
The first relationship for $t \alpha \alpha$ can be readily demonstrated since

$$
\begin{aligned}
F_{t_{t} q_{9}^{2}}= & \frac{1}{3}\left[F_{1}^{t}+F_{2}^{t}+F_{3}^{t}-F_{4}^{t}+2 F_{5}^{t}-2 F_{6}^{t}+2 F_{7}^{t}\right. \\
& \left.-2 F_{8}^{t}-2 F_{9}^{t}+2 F_{10}^{t}-F_{11}^{t}-F_{12}^{t}\right], \quad \text { (B7 }
\end{aligned}
$$

for $i=1,2, \ldots, 6$.

For proving next two relationships for $t \alpha \alpha$ type, we use the method similar to that for s $\alpha \alpha$ type, and obtain

$$
\begin{aligned}
F_{t_{t} q_{20 a}^{2}}=B_{2}= & \frac{1}{12}\left[5 F_{1}^{t}+2 F_{2}^{t}+5 F_{3}^{t}-4 F_{4}^{t}-2 F_{5}^{t}\right. \\
& +10 F_{6}^{t}-8 F_{7}^{t}-2 F_{8}^{t}-2 F_{9}^{t}-2 F_{10}^{t} \\
& \left.+2 F_{11}^{t}-4 F_{12}^{t}\right],
\end{aligned}
$$

for $i=2,5$ and

$$
\begin{aligned}
F_{t_{1} q_{20 a}^{2}}=C_{2}= & \frac{1}{6}\left[F_{1}^{t}+4 F_{2}^{t}+F_{3}^{t}+F_{4}^{t}-4 F_{5}^{t}+2 F_{6}^{t}\right. \\
& +2 F_{7}^{t}-4 F_{8}^{t}-4 F_{9}^{t}-4 F_{10}^{t}+4 F_{11}^{t} \\
& \left.+F_{12}^{t}\right],
\end{aligned}
$$

for $i=1,3,4,6$.

Similarly, for $F_{t_{t} q_{2}^{2}{ }^{2}}$ we have

$$
\begin{aligned}
F_{t_{1} q_{20 b}^{2}}= & \frac{1}{4}\left[F_{1}^{t}+2 F_{2}^{t}+F_{3}^{t}-2 F_{s}^{t}+2 F_{6}^{t}\right. \\
& \left.-2 F_{8}^{t}-2 F_{9}^{t}-2 F_{10}^{t}+2 F_{12}\right],
\end{aligned}
$$

for $i=2,5$, and

$$
F_{t_{i}, 2_{20}^{2}}=\frac{1}{2}\left[F_{1}^{t}+F_{3}^{t}-F_{4}^{t}+2 F_{6}^{t}-2 F_{7}^{t}-F_{12}^{t}\right]
$$

for $i=1,3,4,6$. Combining Eqs. (B9)-(B12) together, we have

$$
\begin{array}{ll}
F_{t \rho_{2}^{2} 20 b}=\frac{1}{3}\left(4 C_{2}-B_{2}\right), & j=2,5 \\
F_{t \rho_{220 b}^{2}}=\frac{1}{3}\left(2 B_{2}+C_{2}\right), & j=1,3,4,6 .
\end{array}
$$
similarly.

Other relations for $F_{t f_{19} q_{20 a}}$ and $F_{t, f_{11} q_{20 b}}$ can be proven

\section{3. $s s \alpha$ and $t$ to types}

The relations of cubic terms between these types can be demonstrated similarly. However, there six are and five in- 
dependent quantities for $s s \alpha$ and $t$ to type, respectively. Therefore more relations, as shown in Table VI, are expected for those types.

Many cross terms $F_{s_{i} s, q}$ or $F_{s, s, q}(i \neq j)$ vanished, some of them do not.

\section{APPENDIX C: THE RELATION BETWEEN SYMMETRIZED COORDINATES AND RIC}

The relations among cubic force constants for benzene in symmetrized coordinates and in RIC are given in Appendices $A$ and $B$, respectively. These two sets of relationships are equivalent. The relation in RIC can be obtained from those in symmetrized coordinates.

For example, from Eqs. (A2) and (A3) we have

$$
q_{20 a}^{2}=\theta_{2}-\frac{1}{\sqrt{3}} \theta_{3}, \quad q_{20 b}^{2}=\theta_{2}+\frac{1}{\sqrt{3}} \theta_{3} \text {. }
$$

Using Eqs. (A9) and (C1) we have

$$
\begin{aligned}
F_{s_{1} q_{200}^{2}} & =F_{s_{1} q_{20}^{+} q_{20}}-\frac{1}{\sqrt{3}} F_{s_{1}\left(q_{20}^{+} q_{20}^{+}+q_{20}^{-} q_{20}\right)} \\
& =B_{1}^{\prime}-\frac{1}{\sqrt{3}} C_{1}^{\prime}, \\
F_{s_{2} q_{20 a}^{2}} & =F_{s_{2} q_{20}^{+} q_{20}^{-}}-\frac{1}{\sqrt{3}} F_{s_{2}\left(q_{20}^{+} q_{20}^{+}+q_{20}^{-} q_{20}\right)} \\
& =B_{1}^{\prime}+\frac{1}{2} \frac{1}{\sqrt{3}} C_{1}^{\prime} .
\end{aligned}
$$

$F_{s_{1} q_{20 a}^{2}}$ and $F_{s_{2} q_{20 a}^{2}}$ are defined as $B_{1}$ and $C_{1}$ in RIC, respectively. Thus,

$$
B_{i}^{\prime}=\frac{1}{3}\left(2 C_{1}+B_{1}\right), \quad C_{1}^{\prime}=\frac{2}{\sqrt{3}}\left(C_{1}-B_{1}\right) .
$$

Using Eqs. (A9), (C1), and (C2) we can obtain Eqs. (4) and (5) given in Table VI:

$$
\begin{aligned}
F_{s_{1} q_{20 b}^{2}} & =F_{s_{1} q_{20}^{+} q_{20}}+\frac{1}{\sqrt{3}} F_{s_{1}\left(q_{20}^{+} q_{20}^{+}+q_{20}^{-} q_{20}^{-}\right)} \\
& =\frac{1}{3}\left(4 C_{1}-B_{1}\right), \\
F_{s_{1} q_{20 b}^{2}} & =F_{s_{2} q_{20}^{+} q_{20}^{-}}+\frac{1}{\sqrt{3}} F_{s_{2}\left(q_{20}^{+} q_{20}^{+}+q_{20} \overline{q_{20}}-\bar{l}\right)} \\
& =\frac{1}{3}\left(C_{1}+2 B_{1}\right) .
\end{aligned}
$$

${ }^{1}$ V. E. Bondybey, Annu. Rev. Phys. Chem. 35, 591 (1984); F. F. Crim, ibid. 35, 657 (1984); E. B. Stechel and E. J. Heller, ibid. 35, 563 (1984); I. Oref and B. S. Rabinovitch, Acc. Chem. Res. 12, 166 (1979); C. S. Parmenter, Faraday Discuss. Chem. Soc. 75, 7 (1983); M. S. Child and L. Halonen, Adv. Chem. Phys. 57, 1 (1984); J. D. McDonald, Annu. Rev. Phys. Chem., 30, 29 (1979); E. L. Sibert III, Int. Rev. Phys. Chem. 9, 1 (1990).

${ }^{2}$ P. J. Robinson and K. A. Holbrook, Unimolecular Reactions (WileyInterscience, New York, 1972); W. Forst, Theory of Unimolecular Reaction (Academic Press, New York, 1973).
${ }^{3}$ S. J. Klippenstein and R. A. Marcus, J. Chem. Phys. 93, 2418 (1990); and references cited there; E. D. Potter, M. Gruebele, L. R. Khundkar, and A. H. Zewail, Chem. Phys. Lett. 164, 463 (1989); and references cited there. ${ }^{4}$ E. g., W. F. Polik, D. R. Guyer, W. H. Miller, and C. B. Moore, J. Chem. Phys. 92, 3471 (1990).

${ }^{5}$ D. S. King, Adv. Chem. Phys. 50, 105 (1982); M. Quack, ibid. 50, 395 (1982).

${ }^{6}$ L. J. Butler, E. J. Hintsa, and Y. T. Lee, J. Chem. Phys. 84, 4104 (1986); A. McIlroy and D. Nesbitt, ibid. 91, 104 (1989).

${ }^{7}$ For example, F. F. Crim, Annu. Rev. Phys. Chem. 35, 657 (1984).

${ }^{8}$ G. Herzberg, Molecular Spectrum and Molecular Structure, Vol. II (Van Nostrand Reinhold, New York, 1945).

${ }^{9}$ E. B. Wilson, Jr., J. C. Decius, and P. C. Cross, Molecular Vibrations (McGraw-Hill, New York, 1955).

${ }^{10}$ S. Brodersen and A. Langseth, Mat. Fys. Skr. Dan. Vid. Selsk. 1, 1 (1956).

${ }^{1}$ K. V. Reddy, D. F. Heller, and M. J. Berry, J. Chem. Phys. 76, 2814 (1982).

${ }^{12}$ S. N. Thakur, L. Goodman, and A. G. Ozkabak, J. Chem. Phys. 84, 6642 (1986).

${ }^{13}$ R. H. Page, Y. R. Shen, and Y. T. Lee, Phys. Rev. Lett. 59, 1293 (1987).

${ }^{14}$ R. H. Page, Y. R. Shen, and Y. T. Lee, J. Chem. Phys. 88, 4621, 5362 (1988).

${ }^{15}$ E. L. Sibert III, J. T. Hynes, and W. P. Reinhardt, J. Chem. Phys. 81, 1135 (1984).

${ }^{16}$ D. H. Lu and W. L. Hase, Chem. Phys. Lett. 142, 187 (1987).

${ }^{17}$ D. H. Lu and W. L. Hase, J. Phys. Chem. 92, 3217 (1988).

${ }^{18}$ K. L. Bintz, D. L. Thompson, and J. W. Brady, J. Chem. Phys. 85, 1848 (1986).

${ }^{19}$ D. L. Clarke and M. A. Collins, J. Chem. Phys. 86, 6871 (1987); 87, 5312 (1987).

${ }^{20}$ L. Halonen, Chem. Phys. Lett. 87, 221 (1982).

${ }^{21}$ E. L. Sibert III, W. P. Reinhardt, and J. T. Hynes, J. Chem. Phys. 81, 1115 (1984).

${ }^{22}$ J. Pliva and A. S. Pine, J. Mol. Spectrosc. 126, 82 (1987).

${ }^{23}$ P. Pulay, G. Fogarasi, and J. E. Boggs, J. Chem. Phys. 74, 3999 (1981).

${ }^{24}$ P. Pulay, J. Chem. Phys. 85, 1703 (1986).

${ }^{25}$ A. G. Ozkabak, L. Goodman, S. N. Thakur, and K. Krogh-Jespersen, J. Chem. Phys. 83, 6047 (1985).

${ }^{26}$ S. N. Thakur, L. Goodman, and A. G. Ozkabak, J. Chem. Phys. 84, 6642 (1986).

${ }^{27}$ A. G. Ozkabak and L. Goodman, J. Chem. Phys. 87, 2564 (1987)

${ }^{28}$ L. Goodman, J. M. Berman, and A. G. Ozkabak, J. Chem. Phys. 90, 2544 (1989).

${ }^{29}$ M. J. Robey and E. W. Schlag, J. Chem. Phys. 67, 2775 (1977).

${ }^{30}$ K. Krogh-Jespersen, R. P. Rava, and L. Goodman, J. Phys. Chem. 88, 5503 (1984)

${ }^{31}$ D. H. Whiffen, Philos. Trans. R. Soc. London, Ser. A 248, 2067 (1971).

${ }^{32}$ J. C. Duinker and 1. M. Mills, Spectrochim. Acta 24A, 417 (1968).

${ }^{33}$ R. A. Kydd, Spectrochim. Acta 27A, 2067 (1971).

${ }^{34}$ S. M. Lederman, S. J. Klippenstein, and R. A. Marcus, Chem. Phys. Lett. 146, 7 (1988); S. M. Lederman and R. A. Marcus, J. Chem. Phys. 88, $6312(1988)$.

${ }^{35}$ For use of an AI search in multiphoton absorption cf, J. V. Tietz and S. I. Chu, Chem. Phys. Lett. 101, 446 (1983); J. Chang and R. E. Wyatt, ibid. 121, 307 (1985); J. Chem. Phys. 85, 1826, 1840 (1986).

${ }^{36}$ J. K. G. Watson, Mol. Phys. 15, 479 (1968); 19, 465 (1970). Our discussion of order is based on a semiclassical analog. Writing a wave function semiclassically as the sum of terms $A \exp (i S / \hbar)$, application of a momentum operator $p$ yields as the leading term $(\partial S / \partial q) A \exp (i S / \hbar)$, whereas application of the momentum operator instead to a function such as $g$ yields $(\hbar / i)(\partial g / \partial q)$ and contains an additional power of $\hbar$. This aspect is discussed later in a paper referred to in Ref. 43.

${ }^{37}$ L. Halonen, T. Carrington, and M. Quack, J. Chem. Soc., Faraday Trans. II 84, 1371 (1988).

${ }^{38}$ J. Pliva, Collect. Czech. Chem. Commun. 23, 777, 1839, 1846 (1958); Z. Chila and J. Pliva, ibid. 28, 1232 (1963).

${ }^{39}$ L. A. Gribov, Opt. Spectrosc. 31,456 (1971).

${ }^{40} \mathrm{R}$. Wallace, Chem. Phys. 11, 181 (1975).

${ }^{41}$ C. R. Quade, J. Chem. Phys. 64, 2783 (1976); 79, 4089 (1983); W. B. Clodius and C. R. Quade, ibid. 80, 3528 (1984); 82, 2365 (1985); C. R. Quade, ibid. 82, 2509 (1985).

${ }^{42}$ E. L. Sibert III, J. T. Hynes, and W. P. Reinhardt, J. Phys. Chem. 87, 2032 (1983).

${ }^{43}$ For CNM the $\chi_{n}^{\prime} s$ are again solutions of a harmonic oscillator equation, 
but are relevant to solutions $\psi_{n}^{\prime}$ of a zeroth-order approximation of Eq. (9) in a form which allows for the curvilinearity of the volume element Jacobian, $g^{1 / 2}, \chi_{n}=g^{1 / 4} \psi_{n}$. This aspect is discussed in a later paper.

${ }^{44}$ P. M. Morse, Phys. Rev. 34, 57 (1929).

${ }^{45}$ G. D. Carney and R. N. Porter, J. Chem. Phys. 65, 3547 (1976).

${ }^{46}$ R. Wallace, Chem. Phys. Lett. 37, 115 (1976).

${ }^{47}$ M. L. Sage, Chem. Phys. 35, 375 (1978).

${ }^{48}$ J. A. C. Gallas, Phys. Rev. A 21, 1829 (1980).

${ }^{49}$ L. Halonen and M. S. Child, Mol. Phys. 46, 239 (1982).

${ }^{50}$ R. H. Tipping and J. F. Ogilvie, J. Chem. Phys. 79, 2537 (1983).

${ }^{51}$ The expression, $\Sigma_{n}\langle l|Q| n\rangle\langle n|Q| m\rangle$, is approximate because the sum only includes the bound levels of the Morse oscillator. Previous work of M. Lewerenz and M. Quack, J. Chem. Phys. 88, 5408 (1988), shows it to be a good approximation for the low lying levels considered here.

${ }^{52}$ Yong-feng Zhang and R. A. Marcus, to be submitted for publication (1990).

${ }^{53}$ W. Meyer, P. Botschwina, and P. G. Burton, J. Chem. Phys. 84, 891 (1986).
${ }^{54}$ S. Carter and N. C. Handy, J. Chem. Phys. 87, 4294 (1987); L. Halonen and T. Carrington, Jr., ibid. 88, 4171 (1988).

${ }^{55}$ A. W. Joshi, Elements of Group Theory for Physicists, 2nd ed. (John Wiley \& Sons, New York, 1977).

${ }^{56}$ Jin-Quan Chen, Group Representation Theory for Physicists (World Scientific, Singapore, 1989).

${ }^{57}$ F. A. Cotton, Chemical Application of Group Theory (Wiley, New York, 1971).

${ }^{58}$ J. P. Elliott and P. G. Dawber, Symmetry in Physics (Oxford University, New York, 1979).

${ }^{59}$ M. Hamermesh, Group Theory and Its Application to Physical Problems (Addison-Wesley, Reading, Massachusetts, 1962).

${ }^{60}$ R. J. Bartlett, S. J. Cole, G. D. Purvis, W. C. Ermler, H. C. Hsieh, and I. Shavitt, J. Chem. Phys. 87, 6579 (1987).

${ }^{61}$ V. K. Wang and J. Overned, Spectrochim. Acta 32A, 1043 (1976).

${ }^{62}$ Y. Bouteiller, M. Allavena, and J. M. Leclercq, J. Chem. Phys. 73, 2851 (1980).

${ }^{63}$ P. Botschwina, Mol. Phys. 3, 729 ( 1976). 